\title{
Hanford Tank Farms Waste Feed Flow Loop Phase VI: PulseEcho System Performance Evaluation
}

\author{
KM Denslow \\ JR Bontha \\ HE Adkins \\ JJ Jenks \\ DF Hopkins
}

November 2012

\section{Pacific Northwest}

NATIONAL LABORATORY

Proudly Operated by Battelle Since 1965 


\title{
DISCLAIMER
}

This report was prepared as an account of work sponsored by an agency of the United States Government. Neither the United States Government nor any agency thereof, nor Battelle Memorial Institute, nor any of their employees, makes any warranty, express or implied, or assumes any legal liability or responsibility for the accuracy, completeness, or usefulness of any information, apparatus, product, or process disclosed, or represents that its use would not infringe privately owned rights. Reference herein to any specific commercial product, process, or service by trade name, trademark, manufacturer, or otherwise does not necessarily constitute or imply its endorsement, recommendation, or favoring by the United States Government or any agency thereof, or Battelle Memorial Institute. The views and opinions of authors expressed herein do not necessarily state or reflect those of the United States Government or any agency thereof.

\author{
PACIFIC NORTHWEST NATIONAL LABORATORY \\ operated by \\ BATTELLE \\ for the \\ UNITED STATES DEPARTMENT OF ENERGY \\ under Contract DE-AC05-76RL01830 \\ Printed in the United States of America \\ Available to DOE and DOE contractors from the \\ Office of Scientific and Technical Information, \\ P.O. Box 62, Oak Ridge, TN 37831-0062; \\ ph: (865) 576-8401 \\ fax: (865) 576-5728 \\ email: reports@adonis.osti.gov \\ Available to the public from the National Technical Information Service, \\ U.S. Department of Commerce, 5285 Port Royal Rd., Springfield, VA 22161 \\ ph: (800) 553-6847 \\ fax: (703) 605-6900 \\ email: orders@ntis.fedworld.gov \\ online ordering: http://www.ntis.gov/ordering.htm
}




\title{
Hanford Tank Farms Waste Feed Flow Loop Phase VI: PulseEcho Sensor Performance Evaluation
}

\author{
KM Denslow \\ JR Bontha \\ HE Adkins \\ JJ Jenks \\ DF Hopkins
}

November 2012

Prepared for

the U.S. Department of Energy

under Contract DE-AC05-76RL01830

Pacific Northwest National Laboratory

Richland, Washington 99352 



\section{Executive Summary}

The delivery of the Hanford double-shell tank waste to the Hanford Tank Waste Treatment and Immobilization Plant (WTP) is governed by specific Waste Acceptance Criteria (WAC) that must be certified as acceptable before any waste can be delivered to the WTP. ICD 19 - Interface Control Document for Waste Feed (Olson 2011) identifies the WTP WAC. Some of the specific WAC pertaining to the waste feed physical and rheological properties are not easily measured with a small sample in an analytical laboratory environment. Critical velocity $\left(\mathrm{V}_{\mathrm{cr}}\right)$ for solids (i.e., the fluid transfer velocity below which pipeline solid particulate deposition occurs) is a key waste acceptance parameter that falls into this category. The ability to detect stationary particles during slurry transfer, a condition directly associated with critical velocity, is the primary focus of this report. Note, although ICD-19 identifies critical velocity as a WAC, it does not define under what conditions a flow is considered to have achieved a "critical velocity”. The definition employed by researchers at the Pacific Northwest National Laboratory (PNNL) encompasses the flow velocity range between the first sign of a pulsatory or a "Stop \& Go" bed to the velocity at which a stationary bed was visually detected.

The current baseline plan of Washington River Protection Solutions (WRPS) ${ }^{1}$ includes a waste feed test loop that will be integrated into the WTP feed delivery systems and will allow real-time measurement of the critical velocity while waste is being circulated through the transfer piping and back to the original source tank. Once critical velocity and other analytically determined acceptance criteria are shown to meet the WAC, the feed will be certified as acceptable for transfer to the WTP receipt tank for further treatment.

During 2010 and 2011, researchers at PNNL conducted an extensive evaluation of the PNNLdeveloped PulseEcho technology for its ability to detect the critical velocity condition of stationary particles in a full-scale, cold-test loop that simulated the WRPS Waste Feed Flow Loop. ${ }^{2}$ The objectives of that work, referred to as Phase III (2010) and Phase IV (2011), was to establish the reliability of the ultrasonic PulseEcho method in detecting stationary particles in the test loop by comparing the flow velocities at which stationary particles were ultrasonically detected and optically/visually detected. ${ }^{3}$ Note, The phases refer to the sequence of project phases (years) that the PulseEcho has been a part of over the years. The results showed excellent agreement between optical/visual detection and ultrasonic detection by the PulseEcho instrument, which demonstrated that the ultrasonic PulseEcho instrument is an excellent candidate for detecting stationary particles in the Waste Feed Flow Loop prior to slurry transfer operations between Hanford tank farms and the WTP. These results are documented in Bontha et al. (2010a, 2010b) and Denslow et al. (2011). The slurries used during the Phase III and Phase IV testing that was conducted at PNNL were designed to possess particle size, density (to a limited extent), and Newtonian/non-Newtonian rheological property values similar to those expected to be encountered during the Hanford tank retrieval operations. The primary objective of these initial work phases was to assess ultrasonic sensor performance and establish measurement reliability. As such, the simulant and carrier fluid combinations were not intended to represent any particular tank waste material.

\footnotetext{
${ }^{1}$ WRPS is the current U.S. Department of Energy contractor for Hanford tank farm operations.

${ }^{2}$ Work done during 2010 also evaluated the PNNL developed Ultrasonic Doppler Velocimeter and Ultrasonic Attenuation methods. PulseEcho was chosen by WRPS as the most suitable technology for further evaluation and eventual application in the Waste Feed Flow Loop.

3 "Optical" indicates visual measurement that is aided by a high resolution camera.
} 
System Performance Testing with the Remote Sampler Demonstration (RSD)/Waste Feed Flow Loop cold-test platform at the Monarch test facility in Pasco, Washington was recently conducted to evaluate the RSD configuration of the Isolok ${ }^{\mathrm{TM}}$ Sampler system, but also afforded an opportunity to continue evaluating the reliability of the ultrasonic PulseEcho instrument. This report summarizes results from PulseEcho testing that was conducted prior to each Isolok ${ }^{\mathrm{TM}}$ demonstration test during the System Performance test campaign. The objective of the PulseEcho System Performance tests was consistent with the objective of prior tests at PNNL; that is, to continue to verify the reliability of the PulseEcho instrument against visual detection of stationary particles. Similar to the testing conducted at PNNL during 2011, two different types of PulseEcho transducers - $5 \mathrm{MHz}$ sensitive to particles $>30 \mu \mathrm{m}$ and $10 \mathrm{MHz}$ sensitive to particles $>14 \mu \mathrm{m}$-were evaluated in the RSD/Waste Feed Flow Loop.

A total of 17 PulseEcho System Performance tests were conducted to evaluate the reliability and repeatability of the PulseEcho system in detecting stationary particles in the RSD/Waste Feed Flow Loop. Unlike the previous testing performed at PNNL, the simulants used in the System Performance tests encompassed particle size, density, and rheological properties of waste feed slurries that are expected to be encountered during Hanford tank waste retrieval operations or bounding for tank waste feed. These simulants were designed to challenge the Isolok ${ }^{\mathrm{TM}}$ Sampler system, but also expanded the simulant test matrix with which the PulseEcho instrument has been tested. In addition to the 17 performance evaluation tests, one performance validation test was conducted to evaluate the long-term repeatability of the PulseEcho measurements. The validation test also helped ensure that electronics recalibration, relocation from PNNL to the Monarch test facility, and the new flow loop design at Monarch did not affect the performance of PulseEcho. The performance validation test utilized a simulant that was used during 2010 and 2011 testing at PNNL.

A summary of the visual and PulseEcho System Performance test results is provided in Table ES.1 in the order in which the tests were planned. The PulseEcho results obtained by the 5-MHz and $10-\mathrm{MHz}$ ultrasonic transducers are highlighted in green or red. Green indicates the PulseEcho instrument detected stationary particles at a flow velocity that is within $\pm 0.3 \mathrm{ft} / \mathrm{sec}$ of the visually determined flow velocities for Regime III ("stop” and "go" bed) and critical velocity V more information on the definitions of the flow behavior observed during critical velocity measurements. Red indicates the PulseEcho instrument detected stationary particles at a flow velocity that is outside \pm 0.3 $\mathrm{ft} / \mathrm{s}$ of the range for Regime III and critical velocity. The flow velocities at which the PulseEcho instrument detected and reported stationary particles in the RSD/Waste Feed Flow Loop are typically within 0.1 to $0.2 \mathrm{ft} / \mathrm{s}$ of the flow velocities at which stationary particles were detected visually/optically. In some cases the PulseEcho instrument detected and reported stationary solids in Regime III, a condition that precedes the formation of a stationary bed of particles and is characterized by transitory stationary particles or pulsatory migration of particle accumulations in the piping. In only one test case, the PulseEcho transducer detected stationary particles at a flow velocity more than $0.3 \mathrm{ft} / \mathrm{s}$ below the range for Regime III and critical velocity. During this test the 5-MHz transducer detected stationary particles within $0.1 \mathrm{ft} / \mathrm{s}$ of the visually determined critical velocity. It is unclear whether the motion detected at the $10-\mathrm{MHz}$ transducer location was a reflection of actual flow conditions at that location or a difference in particle size sensitivity between the two transducer frequencies. 
Table ES.1. Summary of Critical Velocity Detection

\begin{tabular}{|c|c|c|c|c|c|c|c|c|}
\hline \multirow[b]{2}{*}{$\begin{array}{l}\text { Test } \\
\text { Sequence }\end{array}$} & \multicolumn{3}{|c|}{ Target Simulant Properties } & \multirow[b]{2}{*}{$\begin{array}{l}\text { Regime III } \\
\text { Transitory } \\
\text { Settling }\end{array}$} & \multirow{2}{*}{$\begin{array}{c}\text { Visual } V_{\text {cr }} \\
\text { Stationary Bed } \\
\text { of Solids } \\
\text { Upstream and } \\
\text { Downstream }\end{array}$} & \multirow[b]{2}{*}{$\begin{array}{l}\text { PulseEcho } \\
\text { Upstream } \\
\text { (5 MHz) }\end{array}$} & \multirow[b]{2}{*}{$\begin{array}{l}\text { PulseEcho } \\
\text { Downstream } \\
\text { (10 MHz) }\end{array}$} & \multirow[b]{2}{*}{ Visual Observations } \\
\hline & $\begin{array}{c}\text { Base Simulant } \\
\text { Constituents }\end{array}$ & $\begin{array}{c}\text { Supernatant } \\
\text { Simulant } \\
\text { Composition }^{(a)}\end{array}$ & $\begin{array}{l}\text { Base Simulant Mass } \\
\text { Loading/non-Newtonian } \\
\text { Bingham Yield Stress }\end{array}$ & & & & & \\
\hline 46 & Typical & Low & $9 \mathrm{wt} \%$ & Directly to $\mathbf{V}_{\mathbf{c r}}$ & 4.7 & 4.6 & 4.0 & Motion seen upstream at 4.0 and $3.9 \mathrm{ft} / \mathrm{s}$. \\
\hline 32 & Typical & Typical & $9 \mathrm{wt} \%$ & $3.6 \rightarrow 2.7$ & 2.6 & 3.4 & 3.3 & $\begin{array}{l}\text { Sliding piles of solids (dunes) started at } \\
3.4 \mathrm{ft} / \mathrm{s} \text {. Upstream settling at } 2.7 \mathrm{ft} / \mathrm{s} \text {. }\end{array}$ \\
\hline 33 & Typical & High & $9 \mathrm{wt} \%$ & $4.6 \rightarrow 4.3$ & 4.2 & 4.2 & 4.1 & Upstream settling at $4.3 \mathrm{ft} / \mathrm{s}$. \\
\hline 34 & Typical & Low & $13 \mathrm{wt} \%$ & $5.7 \rightarrow 5.2$ & 5.0 & 5.1 & 4.8 & Upstream settling at $5.2 \mathrm{ft} / \mathrm{s}$. \\
\hline 35 & Typical & Typical & $13 \mathrm{wt} \%$ & $3.7 \rightarrow 2.7$ & 2.6 & 3.5 & 3.4 & Sliding piles of solids seen at $3.5 \mathrm{ft} / \mathrm{s}$. \\
\hline 36 & Typical & High & $13 \mathrm{wt} \%$ & $4.8 \rightarrow 4.4$ & 4.3 & 4.4 & 4.3 & Upstream settling at $4.5 \mathrm{ft} / \mathrm{s}$. \\
\hline 37 & High & Low & $9 \mathrm{wt} \%$ & $7.1 \rightarrow 6.9$ & 6.8 & 6.9 & 6.9 & Upstream settling at $7.0 \mathrm{ft} / \mathrm{s}$. \\
\hline 38 & High & Typical & $9 \mathrm{wt} \%$ & $5.4 \rightarrow 5.2$ & 5.1 & 5.3 & 5.0 & Upstream settling at $5.3 \mathrm{ft} / \mathrm{s}$. \\
\hline 39 & High & High & $9 \mathrm{wt} \%$ & $4.4 \rightarrow 4.1$ & 4.0 & 4.1 & 3.9 & Upstream settling at $4.1 \mathrm{ft} / \mathrm{s}$. \\
\hline 40 & High & Low & $13 \mathrm{wt} \%$ & $7.6 \rightarrow 7.1$ & 7.0 & 7.1 & 7.1 & Upstream settling at $7.1 \mathrm{ft} / \mathrm{s}$. \\
\hline 41 & High & Typical & $13 \mathrm{wt} \%$ & $5.6 \rightarrow 5.5$ & 5.4 & 5.4 & 5.3 & Upstream settling at $5.5 \mathrm{ft} / \mathrm{s}$. \\
\hline $41 \mathrm{a}$ & High & Typical & $13 \mathrm{wt} \%$ & $5.9 \rightarrow 5.5$ & 5.4 & 5.5 & 5.4 & Upstream settling at $5.5 \mathrm{ft} / \mathrm{s}$. \\
\hline 42 & High & High & 13 wt $\%$ & $4.5 \rightarrow 4.3$ & 4.2 & 4.3 & 4.1 & Upstream settling at $4.3 \mathrm{ft} / \mathrm{s}$. \\
\hline $42 \mathrm{a}$ & High & High & $13 \mathrm{wt} \%$ & $6.0 \rightarrow 4.2$ & 4.1 & 4.1 & 4.0 & Upstream settling at $4.2 \mathrm{ft} / \mathrm{s}$. \\
\hline 43 & & Non-Newtonian & $3 \mathrm{~Pa}^{\mathrm{a}}$ & $6.0 \rightarrow 5.1$ & 5.0 & 5.4 & $5.1^{(\mathrm{a})}$ & Upstream settling at $5.1 \mathrm{ft} / \mathrm{s}$. \\
\hline 44 & & Non-Newtonian & $10 \mathrm{~Pa}^{\mathrm{a}}$ & $6.8 \rightarrow 5.3$ & 5.2 & 5.7 & $5.3^{(\mathrm{b})}$ & \\
\hline 45 & Typical & Typical & $\begin{array}{l}13 \mathrm{wt} \% \text { ( } 5 \mathrm{wt} \% \text { of the solids } \\
\text { included as spike particles) }\end{array}$ & $3.8 \rightarrow 3.5$ & 3.4 & 3.4 & 3.5 & $\begin{array}{l}\text { Piles of solids settling and eroding at } 3.5 \\
\text { and } 3.6 \mathrm{ft} / \mathrm{s} \text {. }\end{array}$ \\
\hline \multicolumn{9}{|c|}{$\begin{array}{l}\text { (a) The } 10-\mathrm{MHz} \text { transducer did not constantly detect scattering at } 7.2 \mathrm{ft} / \mathrm{s} \text { and reported sediment more than } 10 \% \text { of the time at this flow velocity. } \\
\text { (b) The } 10-\mathrm{MHz} \text { transducer did not constantly detect scattering at } 8.3 \mathrm{ft} / \mathrm{s} \text { and reported sediment more than } 10 \% \text { of the time at this flow velocity. }\end{array}$} \\
\hline \multicolumn{9}{|c|}{ Indicates that PulseEcho instrument detected stationary particles at a flow velocity is within $\pm 0.3 \mathrm{ft} / \mathrm{s}$ of the range for Regime III and $\mathrm{V}_{\mathrm{cr}}$} \\
\hline \multicolumn{9}{|c|}{ Indicates that PulseEcho instrument detected stationary particles at a flow velocity is outside $\pm 0.3 \mathrm{ft} / \mathrm{s}$ of the range for Regime III and $\mathrm{V}_{\mathrm{cr}}$} \\
\hline
\end{tabular}


Although the simulants were not designed to represent actual waste streams encountered during Hanford slurry transfer operations, it is interesting to note from the data in Table ES.1 that only a few slurry simulants formulated for the Isolok ${ }^{\mathrm{TM}}$ System Performance tests had critical flow velocities that were $\leq 4.0 \mathrm{ft} / \mathrm{s}$, and two slurry simulants had critical flow velocities near $7 \mathrm{ft} / \mathrm{s}$. However, differences between the target mass fraction of solids and the actual mass fraction of solids in the slurry simulants that entered the RSD/Waste Feed Flow Loop are expected if the mixing and/or transfer capacity in the test loop were limited. To quantify the actual concentration of the waste stream that passed in front of the PulseEcho transducers during testing, full-diversion samples of 3-4-gal volumes were collected from the RSD/Waste Feed Flow Loop at the beginning and end of every test. Sub-samples of these full-diversion samples are being analyzed for particle concentration and particle size distributions (PSD) by the RJ Lee Group. The data was not available in time to be analyzed and included in this report. Therefore, the scope of this report is limited to reporting the visual test results and the ultrasonic PulseEcho test results from the System Performance test campaign. This report is intended to complement and accompany the report that will be developed by WRPS on the design of the System Performance simulant matrix, the analysis of the full-diversion sample concentration and PSD data, and the design and construction of the RSD/Waste Feed Flow Loop cold-test platform.

Results from the present validation test and results from the 2010 and 2011 test campaigns for the same test simulant are shown in Table ES.2. The data in Table ES.2 show that the flow velocities at which stationary particles were detected by the PulseEcho instrument are highly repeatable and within $\pm 0.3 \mathrm{ft} / \mathrm{sec}$ of the flow velocity at which stationary particles were detected visually during the three-year period over which the PulseEcho system has been evaluated. These results demonstrate the repeatability of PulseEcho measurements and indicate that the instrument was not affected by changes in system configuration.

Table ES.2. Comparison of Validation Test Results from Present and 2010/11 Test Campaigns

\begin{tabular}{|c|c|c|c|c|c|}
\hline \multirow{2}{*}{ Test Campaign } & \multirow{2}{*}{$V_{c r}-$ Visual (ft/s) } & \multicolumn{2}{|c|}{$\mathrm{V}_{\mathrm{cr}}-5 \mathrm{MHz}$ Transducer (ft/s) } & \multicolumn{2}{|c|}{$\mathrm{V}_{\mathrm{cr}}-10 \mathrm{MHz}$ Transducer (ft/s) } \\
\hline & & Half Wall & Full Wall & Half Wall & Full Wall \\
\hline Phase III (2010) & 4.0 & 4.1 & $\mathrm{~N} / \mathrm{A}^{(\mathrm{a})}$ & $\mathrm{N} / \mathrm{A}^{(\mathrm{a})}$ & $\mathrm{N} / \mathrm{A}^{(\mathrm{a})}$ \\
\hline Phase IV (2011) & 4.0 & 4.0 & 4.0 & $\mathrm{~N} / \mathrm{A}^{(\mathrm{a})}$ & 3.9 \\
\hline Phase VI (2012) & 4.0 & $\mathrm{~N} / \mathrm{A}^{(\mathrm{a})}$ & 4.3 & $\mathrm{~N} / \mathrm{A}^{(\mathrm{a})}$ & 4.1 \\
\hline
\end{tabular}

(a) Indicates transducer and/or wall thickness not tested during the particular campaign.

The results from the present testing combined with past PNNL campaigns continue to demonstrate the extremely high reliability of the PulseEcho system to detect the formation of a stationary bed. This, when combined with a measured slurry flow velocity, can be translated into critical velocity for the slurry in question.

All testing performed to date has been conducted under steady-state flow conditions, and PulseEcho measurements have been performed at locations that are 60 to 70 pipe diameters downstream from points of developing flow in the full-scale test loops. These monitoring locations were selected because conditions in the test loops with well-developed flow eliminate uncertainties when assessing PulseEcho performance by comparing with visual measurements. In the actual Tank Farm Waste Feed Flow Loop, a set of two rotating jet mixers will be used to mix the feed vessel and the concentration of the solids drawn out through the transfer pump will vary depending on the location of the jets. Therefore, steady-state conditions in feed concentration cannot be expected to be present as the waste is pumped through the 
Waste Feed Flow Loop. It is not expected that the unsteady solids loading in the feed to the Loop will impact the ability of the PulseEcho instrument to detect stationary particles, but it could impact the methodology used to apply the technology during actual waste feed certification campaigns. In other words, based on the results obtained to date, it is believed that PulseEcho will detect the presence or absence of a stationary bed at the transducer location but translation of the PulseEcho measurement to the appropriate critical velocity will depend on 1) how long and often measurements are made and 2) where the measurements are made. In order to address these two uncertainties in determining critical velocity, how the PulseEcho technique is implemented and used for oscillatory or transient conditions will have to be considered.

The two items identified above-how long and often measurements are made and where the measurements are made - will depend on the mixing and transfer systems used during actual waste feed transfer operations. Therefore, establishing the methodology for implementing the PulseEcho technology during cold testing of the Waste Feed Flow Loop is crucial to actual field deployment of the technology. Such testing can be completed with the verified PulseEcho instrument in its current prototype state. 



\section{Acronyms and Abbreviations}

ASTM

CFM

DAS

DOE

HDI

$\mathrm{Hz}$

ICD

MTEL

NQA

PEEK

PNNL

PSD

PZT

QA

RSD

UDV

UPE

WAC

WRPS

WTP
American Society for Testing and Materials

Coriolis flow meter

data-acquisition system

U.S. Department of Energy

“How Do I” (PNNL’s standards-based management system)

hertz (cycles per second)

interface control document

Multiphase Transport Evaluation Loop

nuclear quality assurance

Polyether ether ketone

Pacific Northwest National Laboratory

particle size distribution

lead zirconate titanate

quality assurance

Remote Sampler Demonstration

Ultrasonic Doppler Velocimeter

Ultrasonic PulseEcho

Waste Acceptance Criteria

Washington River Protection Solutions

Hanford Tank Waste Treatment and Immobilization Plant 



\section{Contents}

Executive Summary .................................................................................................................. ii

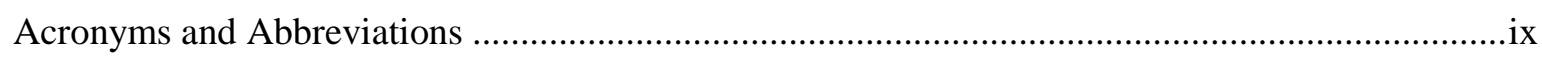

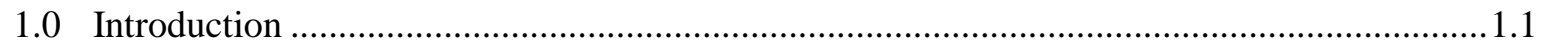

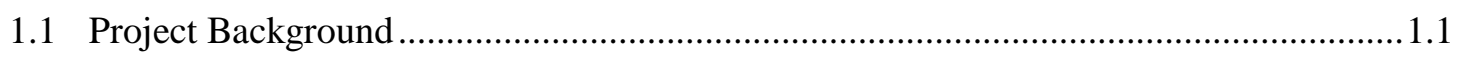

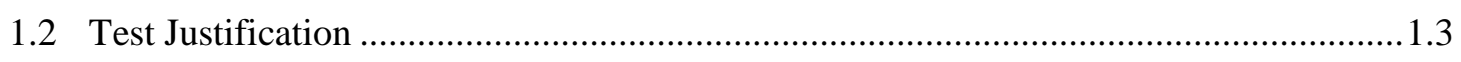

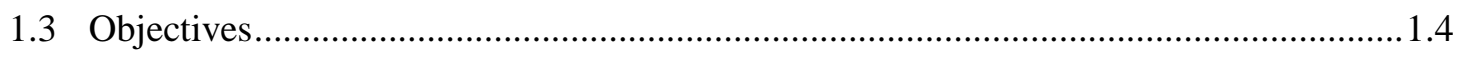

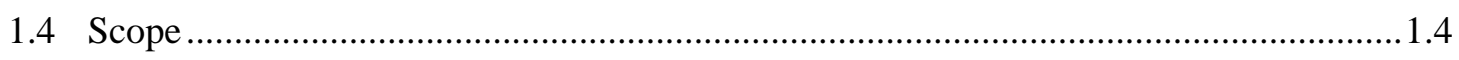

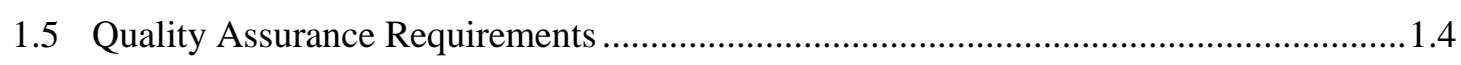

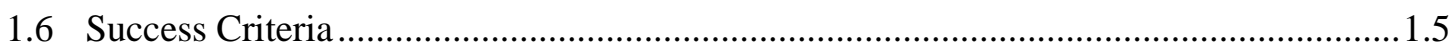

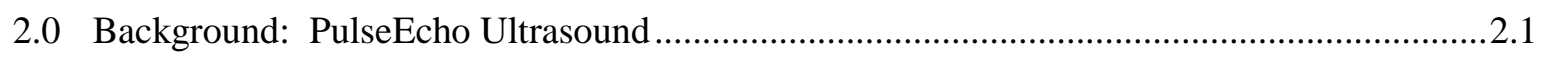

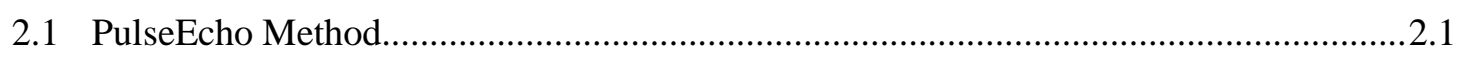

2.1.1 Solids Mobility Detection ….......................................................................2.2

2.1.2 Prior Applications of PulseEcho ….................................................................2.3

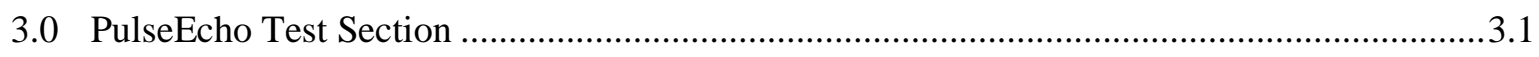

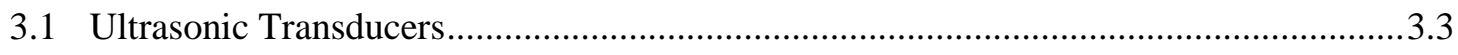

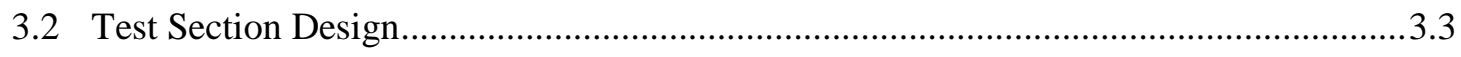

4.0 PulseEcho Measurements and Visual Observations ............................................................

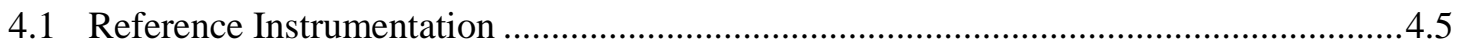

4.2 PulseEcho Configuration......................................................................................

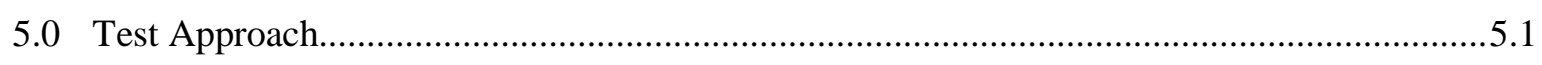

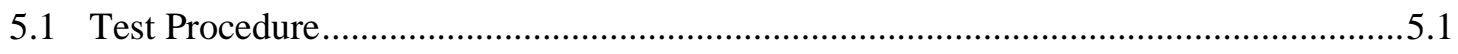

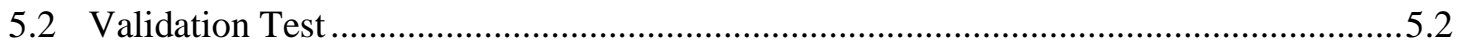

5.3 System Performance Tests .................................................................................... 5.3

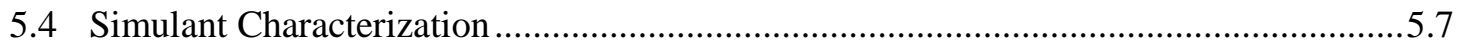

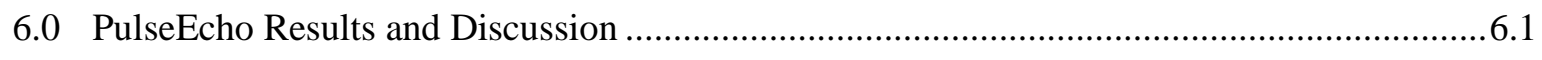

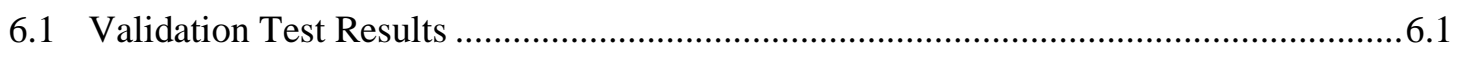

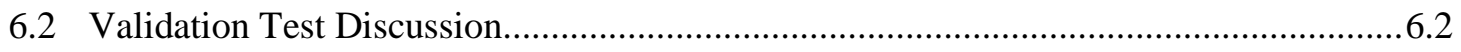

6.3 System Performance PulseEcho Test Results .............................................................. 6.2

6.4 System Performance PulseEcho Discussion ..............................................................

7.0 Considerations for Field Deployment............................................................................. 7.1

7.1 Un-steady State Conditions ................................................................................. 7.1

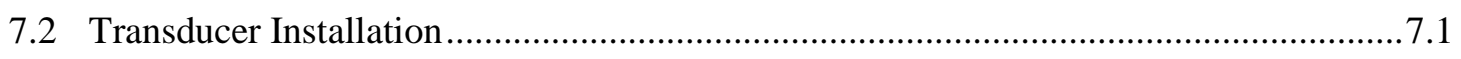

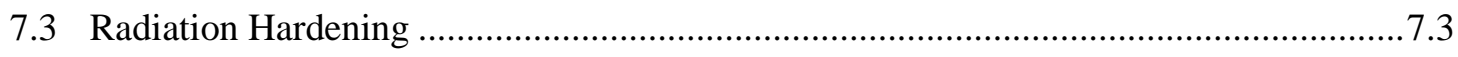

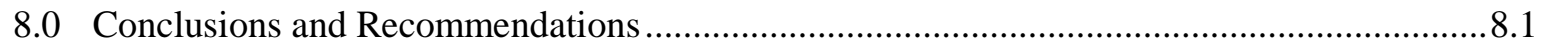

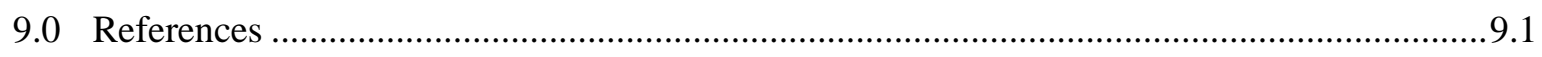

Appendix Test Instruction.................................................................................................... 


\section{Figures}

1.1 Conceptual Illustration of the Double-Shell Tank Waste Feed Certification Process ................ 1.2

2.1 Conceptual Illustration of Ultrasonic Detection of Particle Motion ......................................... 2.2

2.2 Example of an Ultrasonic Backscatter Signal ........................................................................ 2.3

3.1 Photograph of the PulseEcho Instrument Electronics in the Foreground and the Test Section Installations in the Background.............................................................................................

3.2 Photograph of the PulseEcho Test Section in the RSD/Waste Feed Certification Loop ........... 3.2

3.3 Photograph of the High-Resolution Camera Positioned below a Visual Test Sections in the RSD/Waste Feed Certification Loop.................................................................................... 3.2

3.5 PulseEcho Test Section that was Installed in the RSD/Waste Feed Certification Loop ............. 3.3

3.6 Side View of a 10-MHz, 0.25-in.-Diameter Transducer on the Underside of a Water-Filled Schedule 40 Stainless Steel Pipe ......................................................................................... 3.4

4.1 Illustration of PulseEcho Configuration for System Performance Testing................................

6.2 5-MHz, Test Sequence \#35 (13-wt\% Solids) ........................................................................... 6.5

6.3 10-MHz, Test Sequence \#35 (13-wt\% Solids) ......................................................................... 6.5

6.4 5-MHz, Test Sequence \#32 (9-wt\% Solids)......................................................................... 6.6

6.5 10-MHz, Test Sequence \#32 (9-wt\% Solids) ............................................................................ 6.6

7.3 Loosely Embedded Transducer Sound Beam Simulation..................................................... 7.2

7.4 Tightly Embedded Transducer Sound Beam Simulation ....................................................

\section{Tables}

ES.1 Summary of the Visual and PulseEcho Test Results ........................................................... v

ES.2 Comparison of Validation Test Results from Present and 2010/11 Test Campaigns ................ v vii

5.1 Specifications of the Various Particles Used in the Simulant Formulation .............................. 5.3

5.2 Broad PSD Simulant Formulation Used For the Validation Test ............................................ 5.3

5.3 Test Matrix for System Performance Testing in the RSD/Waste Feed Certification Loop ........ $\quad 5.4$

5.4 Test Matrix of Planned System Performance Particle Mixtures and Concentrations ................. 5.4

5.5 Test Matrix of Properties of the System Performance Test Simulant Constituents ................... 5.7

5.6 Test Matrix of Planned System Performance Supernate Mixtures .......................................... 5.7

6.1 Comparison of Validation Test Results from Present and 2010/11 Test Campaigns ................ 6.1

6.2 Summary of Critical Velocity Detection........................................................................................ 


\subsection{Introduction}

This document presents the visual and ultrasonic PulseEcho critical velocity test results obtained from the System Performance test campaign that was completed in September 2012 with the Remote Sampler Demonstration (RSD)/Waste Feed Flow Loop cold-test platform located at the Monarch test facility in Pasco, Washington. This report is intended to complement and accompany the report that will be developed by WRPS on the design of the System Performance simulant matrix, the analysis of the slurry test sample concentration and particle size distribution (PSD) data, and the design and construction of the RSD/Waste Feed Flow Loop cold-test platform.

Section 1.1 describes the background associated with this project. Section 1.2 presents the justification for testing. Section 1.3 lists the overall objectives for this work. Section 1.4 defines the scope of the work for Phase VI. Section 1.5 describes quality assurance (QA) requirements. Section 1.6 lists success criteria.

\subsection{Project Background}

The delivery of Hanford double-shell tank waste to the Hanford Tank Waste Treatment and Immobilization Plant (WTP) will be governed by specific Waste Acceptance Criteria (WAC) that are identified in ICD 19, Interface Control Document for Waste Feed (Olson 2011). Waste must be certified as acceptable before it can be delivered to the WTP. The critical velocity $\left(\mathrm{V}_{\mathrm{cr}}\right)$ is a key waste parameter that must be accurately characterized to determine if the waste is acceptable for transfer to the WTP.

Critical velocity is defined as the fluid transfer velocity at which solid particles begin to deposit on the bottom of a straight horizontal pipe section during slurry transport. The critical velocity depends on the physical properties of the solid particles and carrier fluid and the geometry of the slurry transport system (Oroskar 1980). Critical velocity is not a slurry property that can be directly measured. Instead, the symptoms of critical velocity, chiefly the settling and deposition of solid particles in a pipe, are detected and then correlated with the fluid transfer velocity that resulted in that condition; that is, the critical velocity. The settling and deposition of solid particles in slurry transport piping at the critical velocity are undesirable phenomena during waste-transfer operations to and within the WTP because they are precursors to pipeline plugging that is potentially irreversible. Therefore, the critical velocity of each double-shell tank waste feed must be accurately identified in order to first determine if the waste feed can be accepted by the WTP and then assign a proper fluid transfer velocity above the critical velocity for safe transfer to the WTP.

Washington River Protection Solutions (WRPS), the U.S. Department of Energy contractor for Hanford Tank Operations Contract (TOC), will be responsible for transferring waste from the Hanford double-shell tanks to the WTP via slurry transport piping. WRPS must first certify the waste as acceptable per WAC specified in ICD 19 that were developed to ensure waste feeds can be successfully processed by the WTP. Some of the specific criteria pertaining to the waste feed physical and rheological properties are not easily measured with a small sample in an analytical laboratory environment. The critical velocity in slurry transport piping is a key waste acceptance parameter that falls into this category. The current baseline plan of WRPS is to determine the critical velocity of double-shell tank waste feeds using a Waste Feed Flow Loop. The Waste Certification Loop will be integrated into the WTP feed delivery systems and will allow real-time determination of the critical velocity as waste is being 
circulated through the transfer piping and back to the original source tank as illustrated in Figure 1.1. Once critical velocity and other analytically determined acceptance criteria have been shown to meet the ICD 19 WAC, the waste feed will be certified as acceptable for transfer to the WTP receipt tank for further treatment.

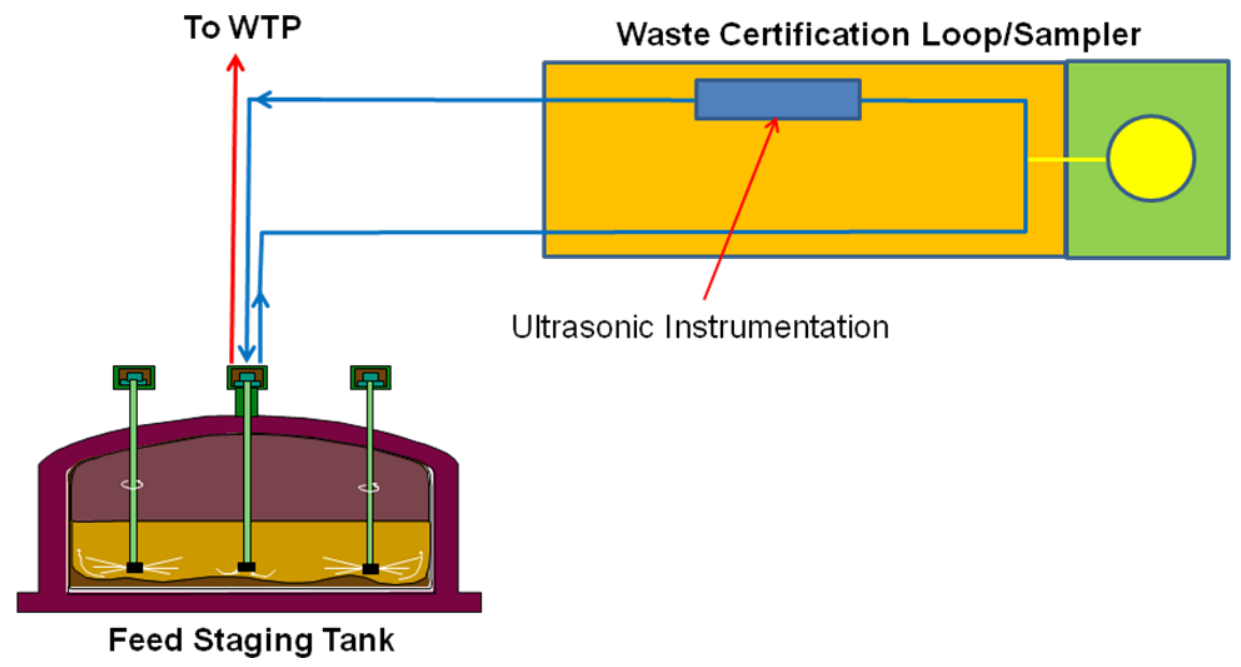

Figure 1.1. Conceptual Illustration of the Double-Shell Tank Waste Feed Test Process

The approach of using a waste feed test loop to determine critical velocity will require real-time monitoring of the test loop piping for particle settling. A method that is sensitive to incipient settling of solid particles will be required to help pinpoint critical velocity and realistically determine if the waste feed can be safely processed by the WTP per ICD 19. Identifying critical velocity with high accuracy will also allow WRPS to assign a proper fluid transfer velocity above the critical velocity during waste transfer to the WTP that will ensure the prevention of solid particulate settling and minimize wear on pumping equipment.

During FY 2009, researchers at Pacific Northwest National Laboratory (PNNL) conducted an extensive review and assessment of currently available instruments and sensors under Phase I and II of the Waste Feed Flow Loop project and selected three ultrasonic instruments-Ultrasonic PulseEcho, Ultrasonic Attenuation, and Ultrasonic Doppler Velocimeter-as the most promising candidates for detecting critical velocity and stationary bed formation in the field-deployed Waste Feed Flow Loop (Meyer et al. 2009). Meyer et al. (2009) included a recommendation for full-scale evaluation of these instruments to establish the reliability of these instruments in detecting critical velocity conditions (stationary particles) and to select one or two of the instruments for further investigation.

During FY 2010, Phase III testing was performed to establish the reliability of these instruments in detecting critical velocity conditions (Bontha et al. 2010a, 2010b). Testing was performed using an existing pipe loop that was originally designed and built to evaluate the pipeline plugging issue during slurry transfer operations at the WTP. The Multiphase Transport Evaluation Loop (MTEL), previously referred to as the "M1-Pipe Loop" at the Process Development Laboratory-East facility at PNNL, was modified to include a test section containing the three instruments being evaluated along with reference instrumentation to facilitate direct comparison of the instrument response with experimentally observed critical velocities. Testing of the ultrasonic sensors was conducted with 3 -in. inner diameter schedule 40 
piping that was operated under typical tank farm, waste-transfer conditions and for a variety of simulated waste streams that were selected to encompass the feed properties of the expected high-level waste.

The results of Phase III testing indicated that both PulseEcho and Ultrasonic Doppler Velocimeter (UDV) are excellent candidates for use in the Waste Feed Flow Loop. The results also indicated that PulseEcho is the more suitable instrument for field deployment. The reason for drawing this conclusion is the PulseEcho system has a distinct advantage over the UDV system in terms of the simplicity in its mounting requirements; the PulseEcho transducer can be mounted on the outside of pipe whereas the UDV system requires breaching the pipe to mount the sensor assembly that includes a material with a sound velocity of approximately $2500 \mathrm{~m} / \mathrm{s}$, such as Rexolite ${ }^{\circledR}$ or polyether ether ketone (PEEK).

During FY 2011, continuing Phase IV testing at the PNNL MTEL facility was performed to evaluate the detection limits of the ultrasonic PulseEcho instrument for a matrix of slurry simulants with low concentrations of small, fast-settling, high-density particles. The results showed that a minimum concentration of stainless steel particles with a PSD d(50) value of 14 micron were required to perform reliable ultrasonic PulseEcho measurements. The minimum required concentration (ranging from 1 - $4 \mathrm{wt} \%$ ) depended on the impact the carrier fluid particles had on the ultrasonic signals.

Phase V testing also was performed during FY 2011 at PNNL to verify the PulseEcho instrument software at its prototype stage. Phases IV and V prepared the PulseEcho instrument for RSD/Waste Feed Flow Loop cold-platform testing that was led by WRPS subcontractor Energy Solutions. The tested PulseEcho spool piece, ultrasonic transducers, PulseEcho instrument, and visual test sections were provided by PNNL to support the FY 2012 System Performance test campaign with the RSD/Waste Feed Flow Loop at the Monarch test facility in Pasco, Washington. The PulseEcho System Performance tests with the RSD/Waste Feed Flow Loop represent Phase VI of PNNL's support to the WRPS waste feed test loop project.

\subsection{Test Justification}

The PulseEcho instrument was used to help determine critical flow velocities of slurry simulants that were selected for System Performance testing with the RSD/Waste Feed Flow Loop cold-test platform. This test campaign was primarily conducted to evaluate the RSD configuration of the Isolok ${ }^{\mathrm{TM}}$ Sampler system, but also afforded an opportunity to continue evaluating the reliability of the ultrasonic PulseEcho instrument with new simulants that were designed to encompassed particle size, density, and rheological properties of waste feed slurries that are expected to be encountered during Hanford tank waste retrieval operations or bounding for tank waste feed. These simulants were not designed to challenge the PulseEcho instrument, as was the case during Phases III and IV, but broadened the database of simulants with which the instrument has been tested to include those slurries expected to be encountered in a "typical” service setting. ${ }^{1}$

\footnotetext{
${ }^{1}$ Challenging simulants for PulseEcho are simulants with particle sizes at the detection range of the transducers i.e., 30 and $14 \mu \mathrm{m}$ for the 5 and $10 \mathrm{MHz}$ transducers, respectively.
} 


\subsection{Objectives}

The objective of the PulseEcho System Performance tests (Phase VI) was consistent with the objective of prior tests at PNNL; that is, to continue to verify the reliability of the PulseEcho instrument against visual detection of stationary particles, but with new simulants that were designed to encompass particle size, density, and rheological properties of waste feed slurries that are expected to be encountered during Hanford tank waste retrieval operations.

\subsection{Scope}

The PulseEcho System Performance tests (Phase VI) included the following activities:

- Delivering the PulseEcho spool piece and the transparent sections to the Monarch test facility for installation in the RSD/Waste Feed Flow Loop cold-test platform

- Developing the test procedure for determining critical flow velocity

- Training Energy Solutions operators to the critical flow velocity test procedure

- Delivering the PulseEcho instrument to the Monarch test facility for System Performance testing

- Operating the PulseEcho instrument during System Performance testing

- Analyzing and reporting the visual and PulseEcho test results.

\subsection{Quality Assurance Requirements}

Under its prime contract with the U.S. Department of Energy (DOE), PNNL's QA Program implements DOE Order 414.1C, Quality Assurance, and 10 CFR 830, Nuclear Safety Management, Subpart A, Quality Assurance Requirements. PNNL has adopted Nuclear Quality Assurance (NQA)1-2000 as its single consensus standard for implementing QA requirements. A graded approach is applied to quality in accordance with NQA-1 Subpart 4.2, Guidance for Graded Application of Quality Assurance for Nuclear-Related Research and Development. PNNL's standards-based management system "How Do I?” (HDI) is its web-based system for communicating the QA Program requirements through laboratorywide procedures or subject areas. All work at PNNL is subject to the applicable requirements of HDI.

Two types of instruments were used-instruments that were part of the PulseEcho system and those that were part of reference measurement used to compare with the PulseEcho data. Instruments that were a part of the PulseEcho system consisted of the transducers and data acquisition hardware. The PulseEcho transducers, which were procured for Phase IV testing and evaluated per American Society for Testing and Materials (ASTM) E1065 Standard Guide for Evaluating Characteristics of Ultrasonic Search Units, were also used for System Performance testing. PulseEcho data-acquisition hardware was calibrated in July 2011, and the annual calibration schedule was extended by three months to allow for the completion of System Performance testing. The PulseEcho software was tested with emulated waveforms to verify that the system and algorithm would detect signal conditions that represent those that result from sediment formation. The reference instruments consisted of a Coriolis mass flow meter to measure the slurry flow rates through the flow loop and visual observations coupled with a highresolution digital camera to observe stationary bed formation in the upstream and downstream visualization sections. The Coriolis mass flow meter was provided by Energy Solutions as part of the 
RSD/Waste Feed Flow Loop instrumentation to acquire enhanced quality data in accordance with ASMENQA-1, Quality Assurance Requirements for Nuclear Facility Applications. The high-resolution camera was provided by PNNL and does not require calibration.

\subsection{Success Criteria}

The success criterion for this project is based on the scope of work listed in Section 1.4. The criterion is the completion of testing to evaluate PulseEcho instrument performance to detect the onset of critical velocity of System Performance simulants that were developed by Energy Solutions to encompass particle size, density, and rheological properties of waste feed slurries that are expected to be encountered during Hanford tank waste retrieval operations. 



\subsection{Background: PulseEcho Ultrasound}

This section presents a brief summary on the principles behind the PulseEcho technology and its past applications. The instrument is described in greater detail by Bontha et al. (2010a, 2010b) and Denslow et al. (2011).

\subsection{PulseEcho Method}

The ultrasonic PulseEcho instrument was developed at PNNL to address the challenges faced by conventional ultrasonic measurement methods in detecting stationary solids (i.e., sediment) under dynamic mixing or flow conditions. The PulseEcho system uses the traditional single-transducer, pulseecho measurement mode; however, the analysis method does not require coherent signal returns in the form of coherent echo patterns from flat, distinct material interfaces that traditional pulse-echo measurement methods rely on. Rather than relying on coherent echo returns to detect interfaces, the PulseEcho system relies on obtaining ultrasonic backscatter energy from an ensemble of sound-scattering particles (a.k.a., “scatterers”).

The instrument's ultrasonic transducer is non-invasively installed on the underside of a vessel or pipe as illustrated in Figure 2.1. The transducer sends ultrasonic pulses through the vessel or pipe wall at energy wavelengths $\lambda$ that are small enough to interact with the solid particles in the slurry. These interactions result in energy scattering, a portion of which is scattered back (i.e., backscattered) in the direction of the transducer. The non-coherent, backscattered energy is detected and recorded in the form of amplitude vs. time signals, where time corresponds with depth in the slurry beyond the pipe or vessel wall via Equation 1.

$$
\mathrm{d}=c(\mathrm{t} / 2)
$$

where $\mathrm{d}=$ depth, $c=$ speed of sound through the stationary particles, and $\mathrm{t}=$ time. The PulseEcho software analyzes the backscatter signals to discriminate between modulated signals (i.e., moving particles) and non-modulated signals (stationary particles) and can also determine where the interface between the two conditions exists to estimate or quantify sediment thickness, if desired. 


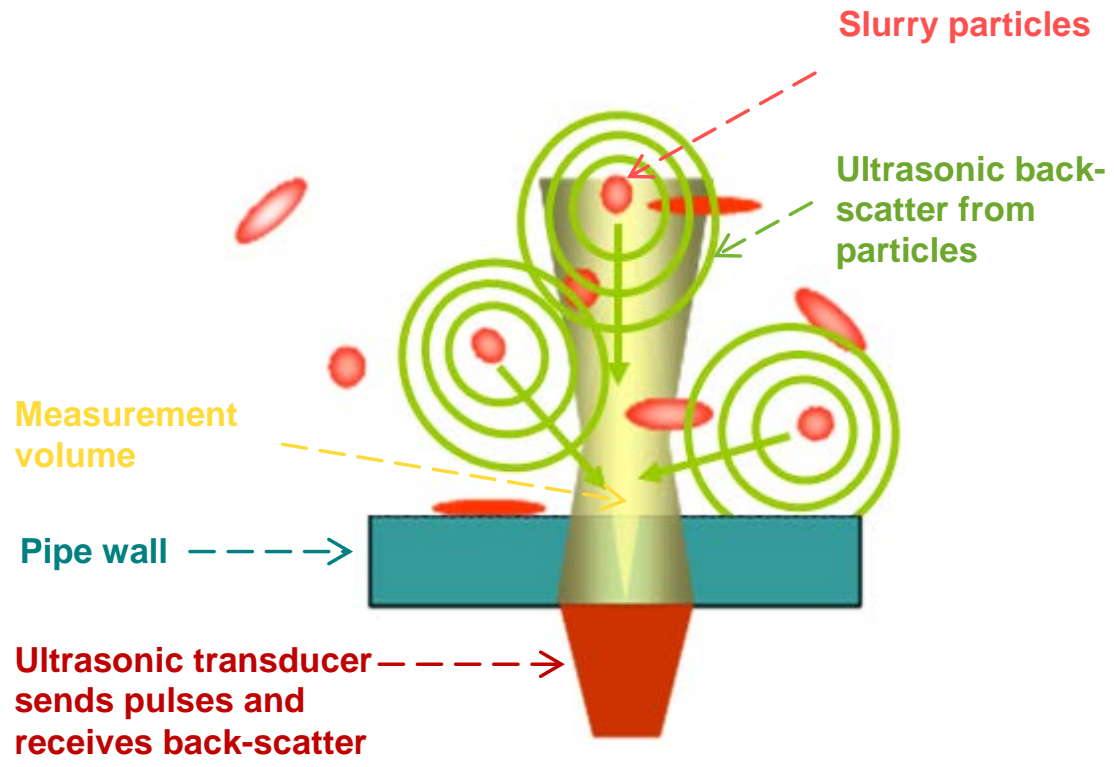

Figure 2.1. Conceptual Illustration of Ultrasonic Detection of Particle Motion

Ultrasonic backscatter is the portion of sound energy that is returned to the transducer after being scattered by reflectors (e.g., glass particles in water). For back-scattering to occur in a fluid, the fluid must contain materials (e.g., particles) with acoustic impedances that are different from those of the surrounding fluid, and the wavelength of ultrasonic energy in the fluid mixture should be on the same order as the sound-scattering material. A minimum particle inventory also must exist in the sound field or insonified fluid volume to generate sufficient backscatter for a reliable measurement. The minimum number of required particles is dependent on the ultrasonic energy wavelength, the size of the sound field, and the size of the particles.

\subsubsection{Solids Mobility Detection}

The PulseEcho instrument uses the backscatter measurement method to detect stationary particles. The user sets the range over which the instrument monitors particle behavior beyond the pipe or vessel wall, and the range-gated, backscatter signals are analyzed by the PulseEcho instrument's variance algorithm to determine if waveforms in the backscatter signals are modulated, signifying particle motion, or not modulated, signifying no particle motion. This particle mobility information is used to determine if solids near the inside wall of the pipe or vessel are completely mobilized, beginning to settle, or stationary/accumulated at the location where the transducer is installed. ${ }^{1}$

\footnotetext{
${ }^{1}$ Although, PulseEcho gives indication regarding particle mobility (or lack of), does not provide any indication regarding nature of the settling particles or which particles settle first.
} 
The measurement volume in the slurry is determined by the transducer geometry, transducer operating frequency and the speed of sound through the slurry. For a round transducer, the measurement volume can be determined by calculating sound beam divergence via Equation 2 and using simple geometry.

$$
\sin (\alpha / 2)=0.514 c / f \mathrm{D}
$$

where $\alpha$ is the beam spread angle of the sound field, $c$ is the speed of sound through the material, $f$ is transducer operating frequency and $\mathrm{D}$ is transducer element diameter.

The PulseEcho instrument typically performs measurements at a rate up to 100 times per second (100 hertz) to keep pace with rapidly changing conditions during flow. Backscatter signals, such as shown in Figure 2.2, are analyzed immediately by the variance algorithm and data on the state of the slurry are presented to the operator in the software user interface. The PulseEcho software automates the measurement process to provide real-time measurements that are presented in a historical graph. Consequently, with these data, the operator can deduce critical flow velocities, characterize the effectiveness of mixing parameters, and quantify the thickness of a stationary layer of solid particles in real time.

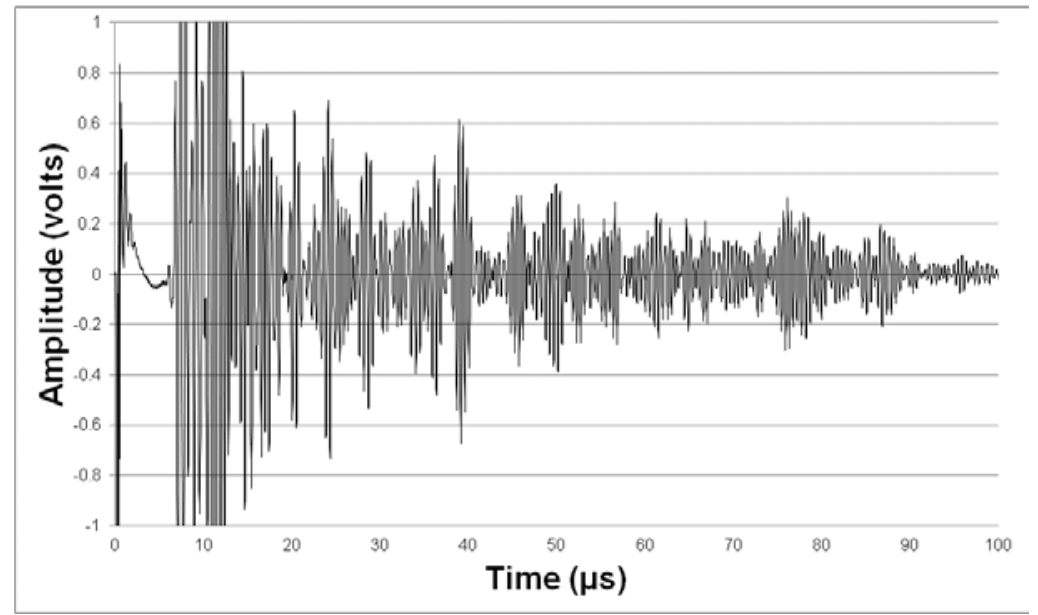

Figure 2.2. Example of an Ultrasonic Backscatter Signal

\subsubsection{Prior Applications of PulseEcho}

The ultrasonic PulseEcho system was developed at PNNL during 2007 and 2008 and was used on the WTP M1 Plugging in Process Piping and M3 Inadequate Design of Mixing Systems-Pulse Jet Mixers projects. The purpose of the PulseEcho system in these applications was to perform non-invasive, realtime ultrasonic detection and measurement of sediment mobility and accumulation in pilot-scale pulse jet mixing vessels and the WTP M1 series initiative test loop (Poloski et al. 2009a, 2009b; Yokuda et al. 2009).

During 2010, WRPS and PNNL began conducting an evaluation of the ability of the ultrasonic PulseEcho instrument to detect stationary particles for the identification of critical velocity in a full-scale waste feed test loop. During 2011, the PulseEcho instrument continued to be evaluated using additional 
Newtonian and non-Newtonian simulants containing small, fast-settling, high-density particles with a mean particle size of $<15 \mu \mathrm{m}$. This two-year evaluation resulted in a verified instrument that has demonstrated the ability to detect stationary particles and identify critical velocity or the onset of critical velocity for a variety of simulated Hanford nuclear waste streams that were selected to encompass expected high-level waste feed properties. These results are documented by Bontha et al. (2010a and 2010b) and Denslow et al. (2011). 


\subsection{PulseEcho Test Section}

The PulseEcho instrument was evaluated at the Monarch test facility in Pasco, Washington. Details related to the test platform will be provided by Energy Solutions who led the design and construction of the RSD/Waste Feed Flow Loop cold-test platform. This section briefly discusses the PulseEcho transducer frequencies and configurations and the PulseEcho test section.

The PulseEcho test section with pre-installed ultrasonic transducers and the two transparent visual test sections were delivered to the Monarch test facility and integrated with the RSD/Waste Feed Flow Loop cold-test platform for System Performance testing. A photograph of the installation is shown in Figure 3.1.

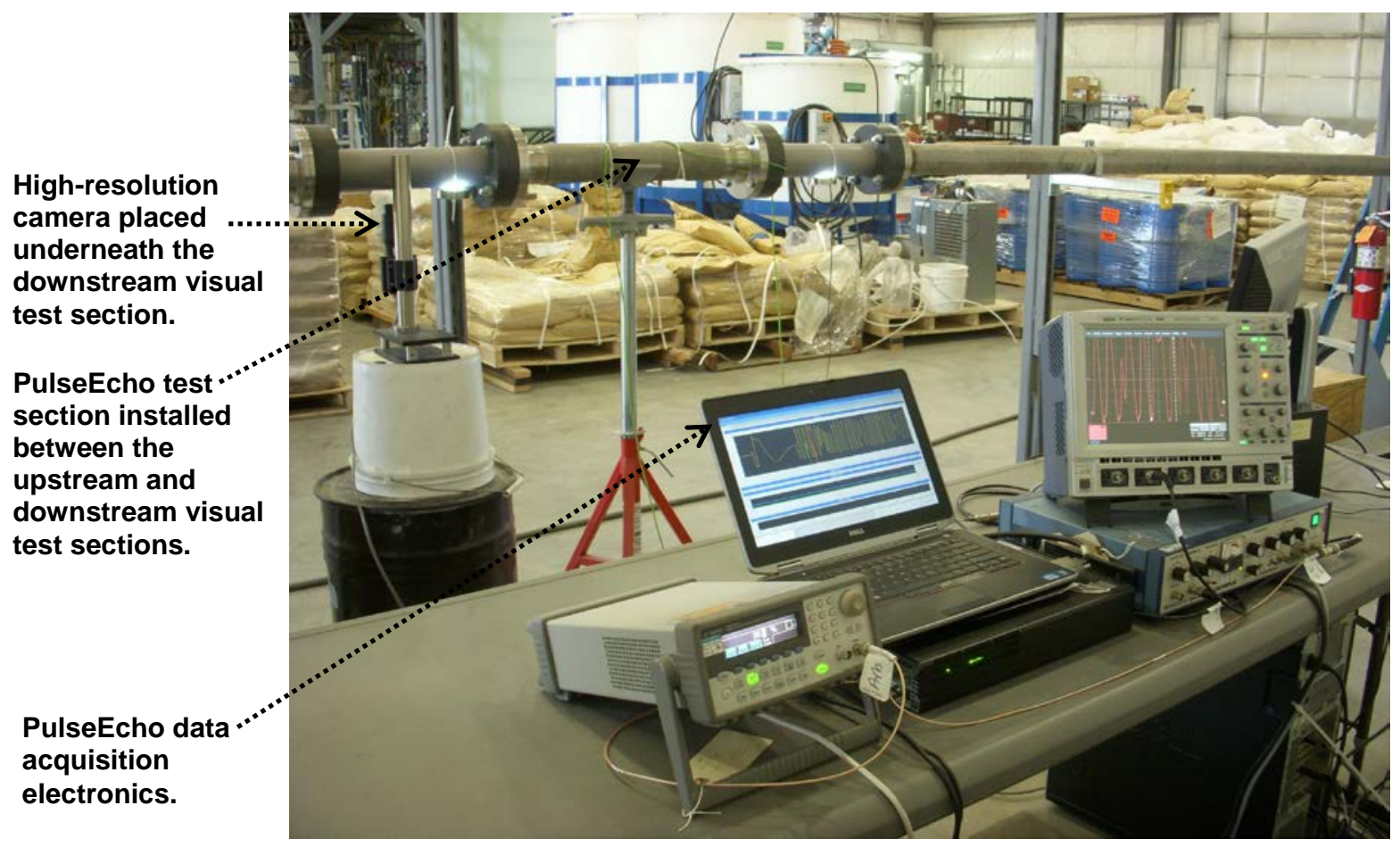

Figure 3.1. Photograph of the PulseEcho Instrument Electronics in the Foreground and the Test Section Installations in the Background. The visual test sections are installed upstream and downstream of the PulseEcho test section in the RSD/Waste Feed Flow Loop. 


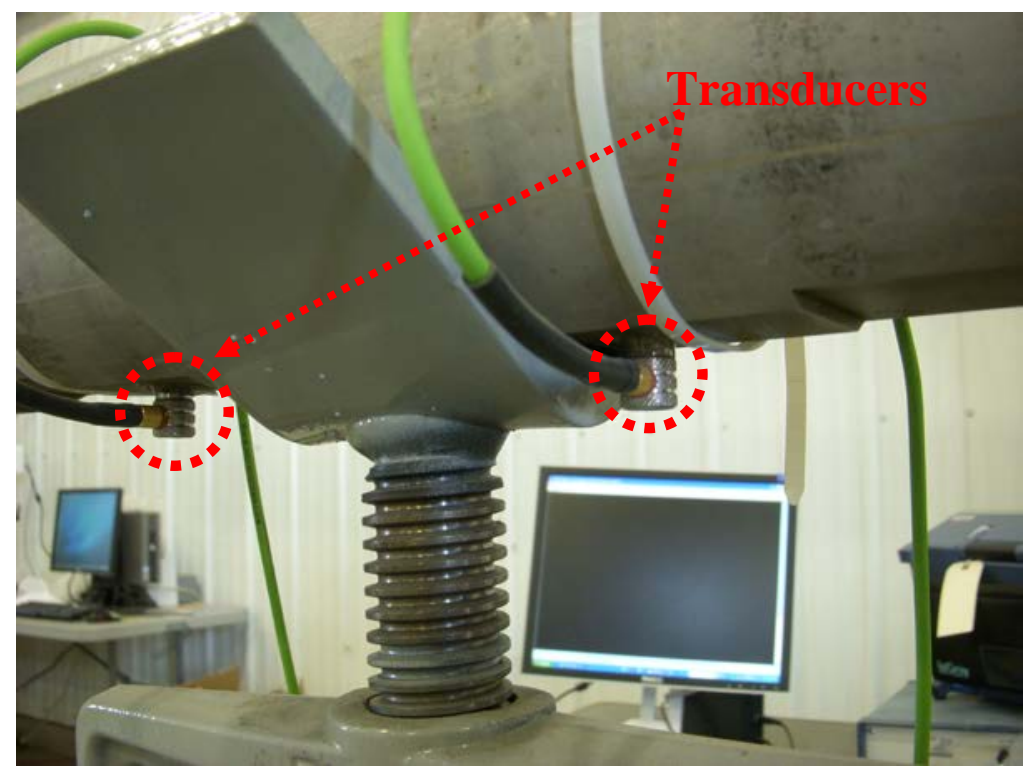

Figure 3.2. Photograph of the PulseEcho Test Section in the RSD/Waste Feed Flow Loop. The 5-MHz and $10-\mathrm{MHz}$ transducers are installed on the underside of the test section.

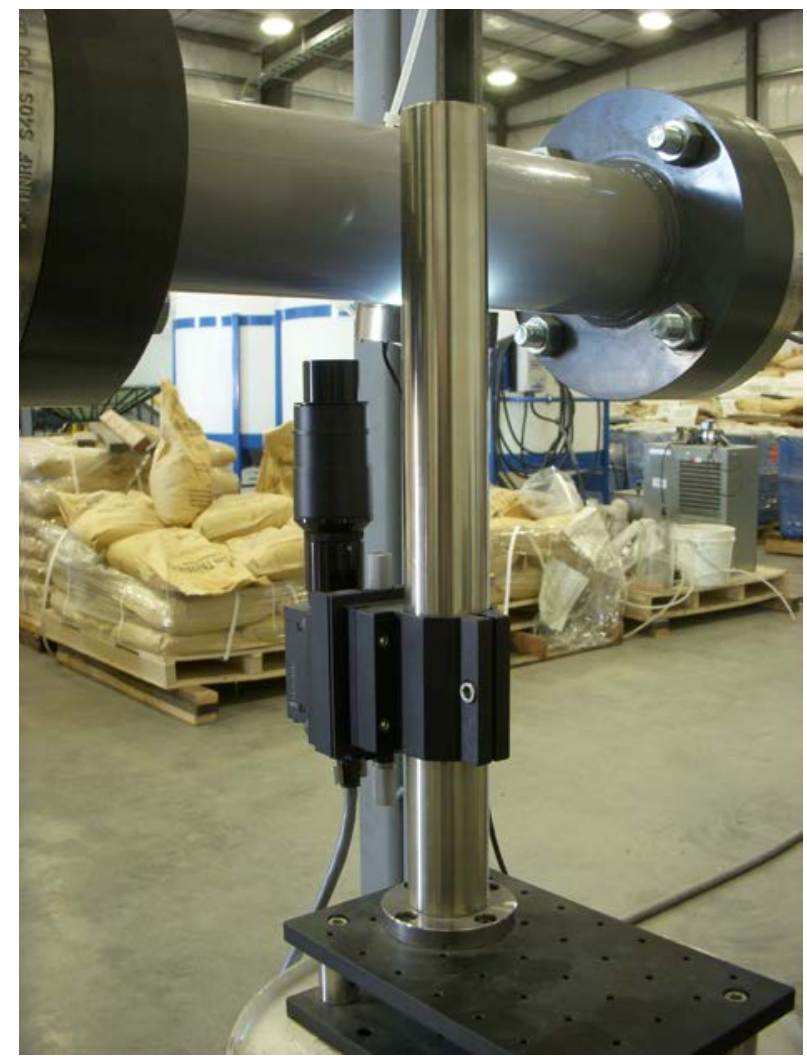

Figure 3.3. Photograph of the High-Resolution Camera Positioned below a Visual Test Sections in the RSD/Waste Feed Flow Loop 


\subsection{Ultrasonic Transducers}

Two PulseEcho transducers with ultrasonic frequencies consistent with those evaluated during Phase III (2010) and Phase IV (2011) at PNNL were evaluated during System Performance testing. These two transducer frequencies were selected based on particle sizes of the simulated waste slurries. The selection process is described in more detail in Denslow et al. (2011). In essence, a 10-MHz transducer was selected for its ability to detect smaller particle sizes of approximately $14 \mu \mathrm{m}$ and larger and a 5-MHz transducer frequency was selected for its ability to detect particle sizes around $30 \mu \mathrm{m}$ and larger. The 10-MHz transducer frequency was first evaluated during Phase IV testing at PNNL, whereas the 5-MHz transducer frequency was evaluated during Phases III and IV. The 5-MHz transducer frequency was used again for System Performance testing to continue to provide continuity across all the test phases.

Contact style ultrasonic transducers were selected for the PulseEcho test section because they allow for the best acoustic impedance matching and thereby maximize sound transmission. Contact style transducers are fabricated with a thin front face plate that serves as an acoustic impedance matching material between the transducer and the test surface and also protects the transducer from damage. The front face plate limits the ultrasonic frequency of the transducer to $10 \mathrm{MHz}$ because its thickness does not allow energy wavelengths smaller than those associated with $\sim 10 \mathrm{MHz}$ to pass. Therefore, a 10-MHz transducer is currently the highest transducer frequency that can be used to effectively transmit ultrasonic energy into the PulseEcho test section for the detection of particles and settling. The contact style transducers that have been used across all three test phases have had 0.25-in.-diameter piezo-composite ultrasonic elements and were purchased from NDT Systems, Inc. (Huntington Beach, California).

\subsection{Test Section Design}

The test section shown in Figure 3.4 was constructed from a 3-in. inner diameter, 0.375-in.-thick wall stainless steel tube and is only approximately 2-ft long. This length allows the transparent visual test sections, which are installed at both ends of the PulseEcho test section, to be relatively closer together. This helps minimize differences in settling behavior observed in the upstream and downstream visual test sections, which is intended to minimize uncertainties of the true settling conditions inside the opaque PulseEcho section. The PulseEcho test section was originally fabricated for Phase IV testing at PNNL for the evaluation of multiple transducer/wall thickness combinations and used again for System Performance testing. A detailed design drawing of the test section can be found in Denslow et al. (2011).

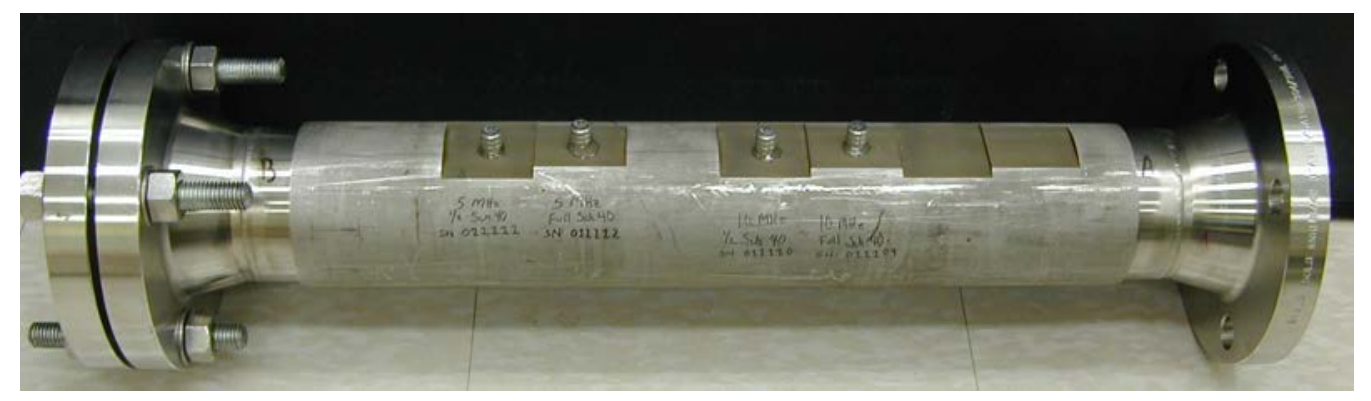

Figure 3.5. PulseEcho Test Section that was Installed in the RSD/Waste Feed Flow Loop. The Test Section is shown with the transducers upright for presentation purposes. 
For each transducer frequency, there are two flats machined at the bottom of the test section such that the thickness is equal to or greater than that of a schedule 40 stainless steel pipe wall (i.e., $\geq 0.216$ in.) or half that of a schedule 40 pipe wall. The flats utilized for System Performance testing with the 5-MHz and $10-\mathrm{MHz}$ transducers were those equal to or greater than that of a schedule 40 stainless steel pipe wall. Each flat is 2-in. long, and the transducer is placed at the center of the flat; this arrangement eliminates any edge effects that may interfere with the path of the ultrasonic signal through the stainless steel. The schedule 40 location machined for the $5-\mathrm{MHz}$ transducer is located $12.6 \mathrm{in}$. downstream of the entry point of the PulseEcho test section, while the schedule 40 location machined for the $10-\mathrm{MHz}$ transducer is located 18.6 in. downstream of the entry point. Therefore, the 5-MHz transducer was located 6 in. upstream of the 10-MHz transducer during System Performance testing. These locations were dictated by the original test section design for Phase IV testing at PNNL.

A simulation of an ultrasonic beam propagating from the front face of the transducer through a schedule 40 pipe wall thickness and into the pipe is shown in Figure 3.6. This simulation was performed using Imagine3D, Version 2.6, a commercial ultrasonic ray tracing software tool developed by UTEX Scientific Instrument, Inc.

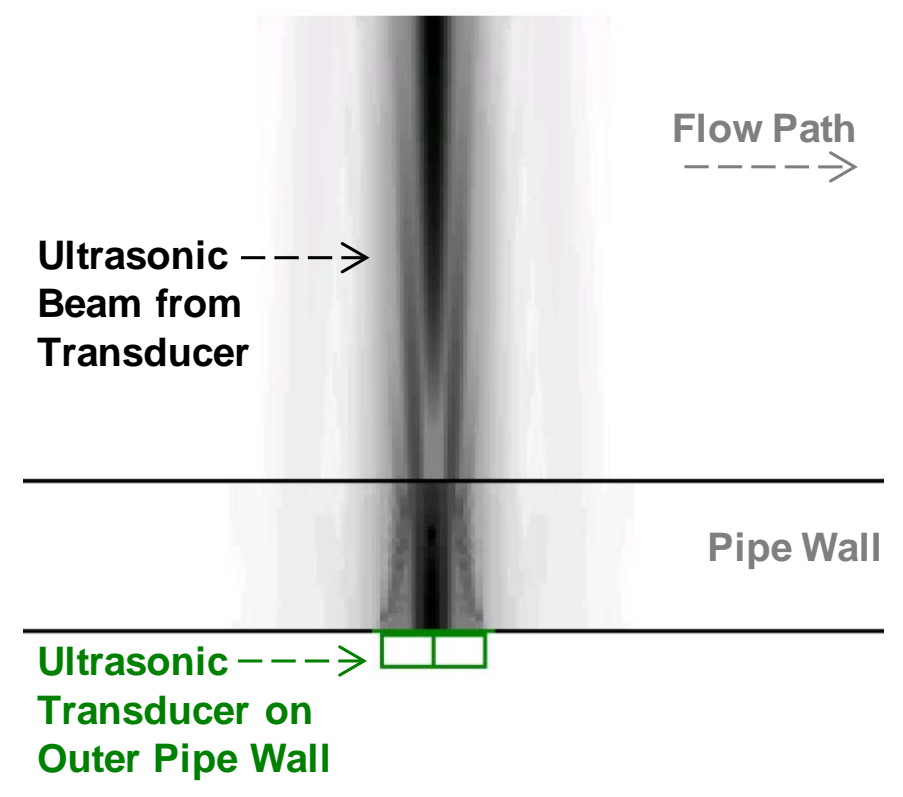

Figure 3.6. Side View of a 10-MHz, 0.25-in.-Diameter Transducer on the Underside of a Water-Filled Schedule 40 Stainless Steel Pipe. The sound field is represented by the black ray traces. 


\subsection{PulseEcho Measurements and Visual Observations}

The objective of the PulseEcho System Performance tests was consistent with the objective of prior tests at PNNL; that is, to continue to verify the reliability of the PulseEcho instrument in detecting stationary particles. This was accomplished by comparing PulseEcho measurements with reference observations made visually and with a high-resolution video camera. This chapter briefly discusses the reference observations and the PulseEcho measurements.

\subsection{Reference Instrumentation}

The pipeline transport of solids suspended in a carrier liquid is considered "critical" when the flow velocity is just at the point where solids suspension becomes challenged. The behavior of the solids at this velocity depends on the specific properties of the solids and the carrier fluid, and may exhibit conditions ranging from a solids concentration gradient, to "saltation," to a "sliding bed," or even a stationary layer of solids. During Phase III, Phase IV and System Performance testing, critical velocity was reported at the velocity at which a stationary bed formed.

Phase III and Phase IV testing at PNNL revealed that the best indication that flow is approaching the critical velocity can be made by using a high-resolution video camera mounted beneath one of the transparent pipe sections. The same approach was used during System Performance testing. The camera is a Point Grey Research model Grasshopper-GRAS20S4M-monochrome (black/white). It has a $1624 \times$ 1224-pixel sensor, with each pixel $4.4 \times 4.4-\mu \mathrm{m}$ square. The camera runs at $30 \mathrm{frames} / \mathrm{second}$ at full resolution $(1600 \times 1200$ pixels). The camera lens is a Donder Zoom Module that provides a field of view of 3200 to $12800 \mu \mathrm{m}$ over the zoom range of the lens. As noted in Bontha et al. (2010a, 2010b), this system is capable of detecting particle behavior from particle sizes ranging from 5 to $500 \mu \mathrm{m}$ in diameter.

\subsection{PulseEcho Configuration}

The PulseEcho electronics and transducer configurations that were employed for System Performance testing were consistent with those used during Phase IV testing at PNNL. The transducers were interfaced with the system of PulseEcho electronics that currently include a waveform generator to provide system timing signals, an ultrasonic pulser/receiver unit to interface with the transducer and transmit and receive ultrasonic signals, and a high-speed analog-to-digital card to convert analog ultrasonic signals to digital signals before sending data to the laptop computer for data analysis and reporting. The digital oscilloscope is used for continuous independent monitoring. A diagram of the data-acquisition electronics configuration is provided in Figure 4.1. 


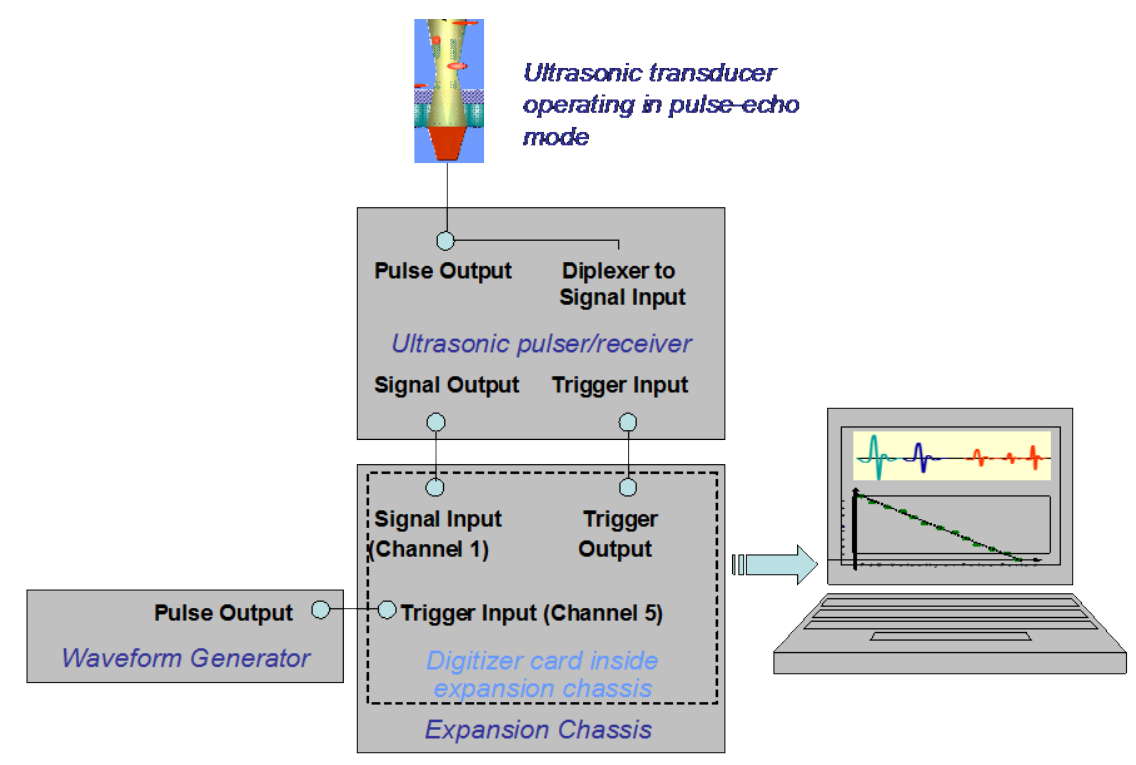

Figure 4.1. Illustration of PulseEcho Configuration for System Performance Testing

Each ultrasonic transducer was operated separately to minimize the risk of undesirable transducer signal crosstalk. Ultrasonic pulses generated by a transducer penetrate the pipe wall and the fluid contents within the pipe. Ultrasonic backscatter signals generated from solid particles contained in the carrier fluid are analyzed and used to determine whether all solids in front of the transducer are mobilized or whether the solids are stationary inside the pipe. Suspended, mobilized solids result in highly modulated ultrasonic backscatter signals with respect to time. Stationary solids result in ultrasonic backscatter signals that are not modulated with respect to time (i.e., wave signals within a stationary solid material repeat consistently, while wave signals from moving particles change with time). The ultrasonic signals received by an ultrasonic transducer are digitized by a digitizer card and analyzed by a computer algorithm. Ultrasonic measurements can be performed on microsecond time scales because sound energy travels on the order of thousands of meters per second through the pipe and slurry. The pulse rates of measurements are limited only by the time required to dissipate energy between pulses. This happens rapidly inside the slurry and allows measurements to be performed at a rate of $100 \mathrm{~Hz}$ or greater. A measurement rate of $100 \mathrm{~Hz}$, which generates 100 signals per second, was selected during System Performance testing. The PulseEcho algorithm uses a set of 10 signals to determine whether stationary solids are present inside the pipe, thus resulting in 10 reports of conditions inside the pipe every second.

PulseEcho measurements were collected over a data-acquisition period of $\geq 2.5$ minutes at each flow velocity under steady-state conditions during System Performance testing. The flow velocities at which the instrument detected settling 10 percent of the time over the acquisition period were reported as critical velocity conditions. This reporting criterion was selected because the onset of critical velocity is typically evidenced by transient particle settling, which results in PulseEcho measurements that fluctuate between zero sediment and detection of sediment. As the flow velocity decreases and the frequency of particle settling increases, the frequency of stationary particle detection increases.

The PulseEcho instrument detects stationary solids; it does not "measure" critical flow velocity. To determine the critical flow velocity, mass flow measurements must be performed and correlated with conditions detected by the PulseEcho instrument. This is done in real time because both instruments 
provide real-time feedback. The RSD/Waste Feed Flow Loop cold-test platform was instrumented with a Coriolis meter by Energy Solutions, which was used to perform the mass flow measurements. Details related to the test platform instrumentation can be found in Energy Solutions document number WI-RSDPR-0002 Rev. 0, WRPS Remote Sampler Demonstration Project - Phase II System Performance Operations Plan. 



\subsection{Test Approach}

PNNL developed the test procedure Test Instructions for Determining Steady State and Critical Velocity that was used to establish the flow conditions for observations and measurements. The test approach used for PulseEcho testing in the RSD/Waste Feed Flow Loop consisted of performing an initial validation test, using a simulant previously tested in the PNNL MTEL loop, before proceeding with the formal System Performance tests planned by Energy Solutions. This section presents the approach as well as the simulants used during the testing.

\subsection{Test Procedure}

The test procedure used for PulseEcho System Performance testing was the same as that previously used during testing at PNNL and presented in Bontha et al. (2010a, 2010b) and Denslow et al. (2011) with the following exceptions:

1. Energy Solutions staff prepared the test loop for each test.

2. Energy Solutions staff prepared and mixed the test simulants.

3. Energy Solutions staff measured the viscosity and density of the test simulants before each test, rather than viscosity before and after each test.

4. Energy Solutions staff loaded the test simulants into the flow loop.

5. Energy Solutions staff operated the test loop, performed and collected flow measurements and density measurements, and determined when steady state was achieved and when data acquisition was to begin.

6. Energy Solutions staff performed and collected temperature measurements.

7. Energy Solutions staff performed and documented visual observations and collected high-resolution camera data during testing per the test procedure located in the Appendix. PNNL provided training for the Energy Solutions operators per the test procedure.

8. Energy Solutions staff collected full-diversion samples of 3-4-gal volumes from the RSD/Waste Feed Flow Loop at the beginning and end of every test for particle concentration and PSD measurements.

9. WRPS required the initial flow velocity for slurry suspension to be $6 \mathrm{ft} / \mathrm{s}$. Higher flow velocities of up to $8 \mathrm{ft} / \mathrm{s}$, the maximum flow velocity capacity of the RSD/Waste Feed Flow Loop cold-test platform, were permitted only if the slurry particles did not suspend at $6 \mathrm{ft} / \mathrm{s}$.

10. Ultrasonic data were collected with only the PulseEcho system. Data collection at each flow velocity involved collecting data with each transducer separately before moving to the next flow velocity. Two measurements were performed at each velocity, one for the 5-MHz transducer and one for the 10-MHz transducer.

Prior to collecting any ultrasonic PulseEcho data on a test simulant, pre-tests were performed to estimate the upper and lower bounds of the flow velocity range that contained the test slurry's critical velocity. This step was necessary in order to reduce the total time duration of the test. The pre-tests were accomplished by setting the flow velocity to $6 \mathrm{ft} / \mathrm{s}$ or, if required, up to $8 \mathrm{ft} / \mathrm{s}$ to fully suspend the slurry particles. The flow velocity was then decreased in 0.5 to $1.0 \mathrm{ft} / \mathrm{s}$ increments, allowing a steady-state condition (characterized by five or more minutes of density measurements within $\pm 0.1 \mathrm{~g} / \mathrm{mL}$ ) to be achieved at each setting. Flow velocity was reduced in this manner until a stationary bed of particles was 
observed in the visual test sections by the Energy Solutions operators. This flow velocity was noted before increasing the flow velocity again to 6 to $8 \mathrm{ft} / \mathrm{s}$ to re-suspend the particles in the slurry. Flow velocity was then reduced to a flow velocity of approximately $1 \mathrm{ft} / \mathrm{s}$ above the point at which a stationary bed of particles had been observed during the pre-tests. After steady state was declared, data were collected with the PulseEcho instrument, visual observations were made, and video was recorded with the high-resolution camera. Data were collected with each transducer over a period of $\geq 2.5$ minutes at a measurement rate of $100 \mathrm{~Hz}$. Flow velocity was decreased in increments of 0.1 to $0.2 \mathrm{ft} / \mathrm{s}$ and data collected at each increment after steady state was reached. This was repeated until the PulseEcho instrument detected stationary particles. The test procedure that was followed by the operators is located in the appendix.

\subsection{Validation Test}

An initial validation test was performed in the RSD/Waste Feed Flow Loop using a simulant containing 20 -wt $\%$ glass beads in water. The glass bead simulant was composed of a broad distribution of particles sizes with the same density of $2.50 \mathrm{~g} / \mathrm{mL}$. This simulant is considered to be high in solids concentration in a carrier fluid with low viscosity and low yield stress. The broad PSD formulation and the property and supplier information for the glass particle constituents are provided in Table 5.1 and Table 5.2. The density of the particles presented are the nominal values and the $\mathrm{d}(50)$ particle size is based on the volume fraction.

The three purposes for performing the validation test in the RSD/Waste Feed Flow Loop are described below:

1. To determine if the visually determined critical velocity in the RSD/Waste Feed Flow Loop cold-test platform was consistent with the critical velocity that had been determined during previous Phases III and IV testing at PNNL for the same simulant

2. To determine if the PulseEcho transducers detected settling at the same flow velocities for the same simulant

3. To train Energy Solutions operators to the critical velocity procedure developed by PNNL.

The validation test was performed with PNNL operators present who had performed Phase III and IV testing at PNNL. The PNNL operators worked with three Energy Solutions operators to practice the test procedure and perform the validation test. The cross-training of staff was performed once more for the first formal System Performance test because this test occurred approximately four months after the validation test. 
Table 5.1. Specifications of the Various Particles Used in the Simulant Formulation

\begin{tabular}{|c|c|c|c|c|}
\hline Simulant Name & Supplier/Manufacturer & Product ID & $\begin{array}{l}\text { Density } \\
\text { (g/mL) }\end{array}$ & $\begin{array}{l}\text { Particle Size } \\
\text { d(50), } \mu \mathrm{m}\end{array}$ \\
\hline SPHERIGLASS® 5000 & Potters Industries & A Glass, 5000 & 2.50 & 7.1 \\
\hline SPHERIGLASS® 3000 & Potters Industries & A Glass, 3000 & 2.50 & 34.0 \\
\hline BALLOTINI Mil \#13 & Potters Industries & MIL-PRF-9954D\#13 & 2.50 & 57.7 \\
\hline BALLOTINI Mil \#10 & Potters Industries & MIL-PRF-9954D\#10 & 2.50 & 114.9 \\
\hline BALLOTINI Mil\#8 & Potters Industries & MIL-PRF-9954D\#11 & 2.50 & 177.4 \\
\hline BALLOTINI Mil \#6 & Potters Industries & MIL-PRF-9954D\#6 & 2.50 & 190.5 \\
\hline BALLOTINI Mil \#4 sieved <500 $\mu \mathrm{m}$ & Potters Industries & MIL-PRF-9954D\#4 & 2.50 & 502.8 \\
\hline
\end{tabular}

Table 5.2. Broad PSD Simulant Formulation Used For the Validation Test

\begin{tabular}{|c|c|c|c|c|}
\hline Simulant Name & Composition & $\begin{array}{l}\text { Component } \\
\text { (wt \%) }\end{array}$ & $\begin{array}{l}\text { Density } \\
(\mathrm{g} / \mathrm{mL})\end{array}$ & $\begin{array}{c}\text { Particle Size (volume) } \\
\text { d(50), } \mu \mathrm{m}\end{array}$ \\
\hline \multirow{6}{*}{ Broad PSD } & SPHERIGLASS ${ }^{\circledR} 5000$ & 7 & \multirow{6}{*}{2.50} & \multirow{6}{*}{93.8} \\
\hline & SPHERIGLASS $® 3000$ & 14 & & \\
\hline & BALLOTINI Mil \#13 & 29 & & \\
\hline & BALLOTINI Mil \#10 & 29 & & \\
\hline & BALLOTINI Mil \#6 & 14 & & \\
\hline & BALLOTINI Mil \#4 & 7 & & \\
\hline
\end{tabular}

\subsection{System Performance Tests}

The planned slurry simulant matrix that was designed by WRPS for System Performance testing is presented in Table 5.3. ${ }^{1}$ The planned simulant constituents for each test and their properties are presented in Table 5.4 through Table 5.6. Additional details on these simulants can be found in Energy Solutions document number WI-RSD-PR-0002 Rev. 0, WRPS Remote Sampler Demonstration Project - Phase II System Performance Operations Plan.

The System Performance slurry simulant matrix was designed to evaluate the ability of the RSD Isolok ${ }^{\mathrm{TM}}$ Sampler system to obtain reliable samples from the RSD/Waste Feed Flow Loop. These simulants were not designed to challenge the PulseEcho instrument, as was the case during Phase III and IV testing at PNNL, but broadened the database of simulants with which the instrument has been tested to include those slurries expected to be encountered in a "typical" service setting.

\footnotetext{
${ }^{1}$ Details of the test simulants are discussed in detail in RPP-PLAN-52623, Rev A, One System Waste Feed Delivery Mixing and Sampling Program System Performance Test Plan, WRPS. 2012
} 
Table 5.3. Test Matrix for System Performance Testing in the RSD/Waste Feed Flow Loop

\begin{tabular}{|c|c|c|c|}
\hline $\begin{array}{c}\text { Test } \\
\text { Sequence }\end{array}$ & $\begin{array}{c}\text { Base Simulant } \\
\text { Constituents }\end{array}$ & $\begin{array}{l}\text { Supernatant Simulant } \\
\text { Composition (a) }\end{array}$ & $\begin{array}{l}\text { Base Simulant Mass Loading / non- } \\
\text { Newtonian Bingham Yield Stress }\end{array}$ \\
\hline 32 & Typical & Typical & $9 \mathrm{wt} \%$ \\
\hline 33 & Typical & High & $9 \mathrm{wt} \%$ \\
\hline 34 & Typical & Low & $13 \mathrm{wt} \%$ \\
\hline 35 & Typical & Typical & $13 \mathrm{wt} \%$ \\
\hline 36 & Typical & High & $13 \mathrm{wt} \%$ \\
\hline 37 & High & Low & 9 wt $\%$ \\
\hline 38 & High & Typical & $9 \mathrm{wt} \%$ \\
\hline 39 & High & High & 9 wt $\%$ \\
\hline 40 & High & Low & $13 \mathrm{wt} \%$ \\
\hline $41 \& 41 a$ & High & Typical & $13 \mathrm{wt} \%$ \\
\hline $42 \& 42 a$ & High & High & $13 \mathrm{wt} \%$ \\
\hline 43 & & Non-Newtonian & $3 \mathrm{~Pa}^{(\mathrm{a})}$ \\
\hline 44 & & Non-Newtonian & $10 \mathrm{~Pa}^{(\mathrm{a})}$ \\
\hline 45 & Typical & Typical & $13 \mathrm{wt} \%$ with $5 \mathrm{wt} \%$ added as spike particles \\
\hline 46 & Typical & Low & 9 wt\% \\
\hline
\end{tabular}

Table 5.4. Test Matrix of Planned System Performance Particle Mixtures and Concentrations

\begin{tabular}{ll}
\hline Test Sequence 32 & Mass wt\% \\
\hline Small Gibbsite & $2.43 \%$ \\
Large Gibbsite & $3.96 \%$ \\
Medium Sand & $1.17 \%$ \\
Zirconium Oxide & $0.90 \%$ \\
Stainless Steel & $0.5 \%$ \\
Supernatant (Typical) & $91.0 \%$ \\
\hline Test Sequence 33 & Mass wt\% \\
\hline Small Gibbsite & $2.43 \%$ \\
Large Gibbsite & $3.96 \%$ \\
Medium Sand & $1.17 \%$ \\
Zirconium Oxide & $0.90 \%$ \\
Stainless Steel & $0.5 \%$ \\
Supernatant (High) & $91.0 \%$ \\
\hline
\end{tabular}


Table 5.4. (contd)

\begin{tabular}{|c|c|}
\hline Test Sequence 34 & Mass wt \% \\
\hline Small Gibbsite & $3.51 \%$ \\
\hline Large Gibbsite & $5.72 \%$ \\
\hline Medium Sand & $1.69 \%$ \\
\hline Zirconium Oxide & $1.30 \%$ \\
\hline Stainless Steel & $0.8 \%$ \\
\hline Supernatant (Low) & $87.0 \%$ \\
\hline Test Sequence 35 & Mass wt \% \\
\hline Small Gibbsite & $3.51 \%$ \\
\hline Large Gibbsite & $5.72 \%$ \\
\hline Medium Sand & $1.69 \%$ \\
\hline Zirconium Oxide & $1.30 \%$ \\
\hline Stainless Steel & $0.8 \%$ \\
\hline Supernatant (Typical) & $87.0 \%$ \\
\hline Test Sequence 36 & Mass wt \% \\
\hline Small Gibbsite & $3.51 \%$ \\
\hline Large Gibbsite & $5.72 \%$ \\
\hline Medium Sand & $1.69 \%$ \\
\hline Zirconium Oxide & $1.30 \%$ \\
\hline Stainless Steel & $0.8 \%$ \\
\hline Supernatant (High) & $87.0 \%$ \\
\hline Test Sequence 37 & Mass wt\% \\
\hline Large Gibbsite & $0.27 \%$ \\
\hline Small Sand & $3.15 \%$ \\
\hline Large Sand & $1.89 \%$ \\
\hline Zirconium Oxide & $0.72 \%$ \\
\hline Stainless Steel & $3.0 \%$ \\
\hline Supernatant (Low) & $91.0 \%$ \\
\hline Test Sequence 38 & Mass wt\% \\
\hline Large Gibbsite & $0.27 \%$ \\
\hline Small Sand & $3.15 \%$ \\
\hline Large Sand & $1.89 \%$ \\
\hline Zirconium Oxide & $0.72 \%$ \\
\hline Stainless Steel & $3.0 \%$ \\
\hline Supernatant (Typical & $91.0 \%$ \\
\hline Test Sequence 39 & Mass wt\% \\
\hline Large Gibbsite & $0.27 \%$ \\
\hline Small Sand & $3.15 \%$ \\
\hline Large Sand & $1.89 \%$ \\
\hline Zirconium Oxide & $0.72 \%$ \\
\hline Stainless Steel & $3.0 \%$ \\
\hline Supernatant (High) & $91.0 \%$ \\
\hline Test Sequence 40 & Mass wt\% \\
\hline Large Gibbsite & $0.39 \%$ \\
\hline Small Sand & $4.55 \%$ \\
\hline Large Sand & $2.73 \%$ \\
\hline Zirconium Oxide & $1.04 \%$ \\
\hline Stainless Steel & $4.3 \%$ \\
\hline Supernatant (Low) & $87.0 \%$ \\
\hline
\end{tabular}


Table 5.4. (contd)

\begin{tabular}{|c|c|}
\hline Test Sequence 41 & Mass wt \% \\
\hline Large Gibbsite & $0.39 \%$ \\
\hline Small Sand & $4.55 \%$ \\
\hline Large Sand & $2.73 \%$ \\
\hline Zirconium Oxide & $1.04 \%$ \\
\hline Stainless Steel & $4.3 \%$ \\
\hline Supernatant (Typical) & $87.0 \%$ \\
\hline Test Sequence 42 & Mass wt\% \\
\hline Large Gibbsite & $0.39 \%$ \\
\hline Small Sand & $4.55 \%$ \\
\hline Large Sand & $2.73 \%$ \\
\hline Zirconium Oxide & $1.04 \%$ \\
\hline Stainless Steel & $4.3 \%$ \\
\hline Supernatant (High) & $87.0 \%$ \\
\hline Test Sequence 43 and 44 & Mass wt \% \\
\hline Zirconium Oxide & $1.16 \%$ \\
\hline Stainless Steel & $4.78 \%$ \\
\hline Non-Newtonian 3 and $10 \mathrm{~Pa}$ & $94.06 \%$ \\
\hline Test Sequence 45 & Mass wt \% \\
\hline Small Gibbsite & $3.33 \%$ \\
\hline Large Gibbsite & $5.43 \%$ \\
\hline Medium Sand & $1.61 \%$ \\
\hline Zirconium Oxide & $1.24 \%$ \\
\hline Stainless Steel & $0.74 \%$ \\
\hline Stainless Steel 1/16" & $0.65 \%$ \\
\hline Supernatant (Typical) & $87.00 \%$ \\
\hline Test Sequence 46 & Mass wt \% \\
\hline Small Gibbsite & $2.41 \%$ \\
\hline Large Gibbsite & $3.95 \%$ \\
\hline Medium Sand & $1.15 \%$ \\
\hline Stainless Steel & $0.92 \%$ \\
\hline Zirconium Oxide & $0.52 \%$ \\
\hline Supernatant (Typical) & $91.00 \%$ \\
\hline Test Sequence 41a & Mass wt\% \\
\hline Large Gibbsite & 0.38 \\
\hline Small Sand & $4.55 \%$ \\
\hline Large Sand & $2.75 \%$ \\
\hline Zirconium Hydroxide & $1.04 \%$ \\
\hline Stainless Steel & $4.31 \%$ \\
\hline Supernatant (Typical) & $87.00 \%$ \\
\hline Test Sequence 42a & Mass wt \% \\
\hline Large Gibbsite & $0.40 \%$ \\
\hline Small Sand & $4.56 \%$ \\
\hline Large Sand & $2.73 \%$ \\
\hline Zirconium Oxide & $1.03 \%$ \\
\hline Stainless Steel & $4.30 \%$ \\
\hline Supernatant (High) & $87.00 \%$ \\
\hline
\end{tabular}


Table 5.5. Test Matrix of Properties of the System Performance Test Simulant Constituents

\begin{tabular}{lccc}
\hline Simulant & SG & Density (lbs/ft $\left.\mathbf{3}^{\mathbf{}}\right)$ & Particle Size (d50, um) \\
\hline Small Gibbsite & 2.42 & 151.008 & 1.3 \\
Large Gibbsite & 2.42 & 151.008 & 10 \\
Small Sand & 2.65 & 165.36 & 57 \\
Medium Sand & 2.65 & 165.36 & 148 \\
Large Sand & 2.65 & 165.36 & 382 \\
Zirconium Oxide & 5.7 & 355.68 & 6 \\
Stainless Steel & 8 & 499.2 & 112 \\
Stainless Steel 1/16" & 8 & 499.2 & 1587 \\
Kaolin & 2.68 & 167.232 & 1.02 \\
Sodium Thiosulfate & 1.667 & 104.0208 & N/A \\
Glycerol & 1.26 & 78.624 & N/A \\
Water & 1 & 62.4 & N/A \\
Low & 1.1 & 68.64 & N/A \\
High & 1.37 & 85.488 & N/A \\
Typical & 1.29 & 80.496 & N/A \\
Non-Newtonian 3 and 10 Pa & 1.2 & 74.88 & N/A \\
Non-Newtonian 3 and 10 Pa & 1.37 & 85.488 & \\
\hline
\end{tabular}

Table 5.6. Test Matrix of Planned System Performance Supernate Mixtures

\begin{tabular}{cccc}
\hline Supernate & Glycerol & Sodium Thiosulfate & Kaolin \\
\hline Low & $0.0 \%$ & $12.0 \%$ & $0.0 \%$ \\
Typical & $0.0 \%$ & $31.5 \%$ & $0.0 \%$ \\
High & $19.5 \%$ & $33.4 \%$ & $0.0 \%$ \\
$3 \mathrm{~Pa}$ & $0.0 \%$ & $0.0 \%$ & $22.0 \%$ \\
$10 \mathrm{~Pa}$ & $0.0 \%$ & $0.0 \%$ & $28.0 \%$
\end{tabular}

(a) The percentages represent the relative amounts (by wt) of constituents listed with the remainder made up with water

\subsection{Simulant Characterization}

Full-diversion samples of 3-4-gal volumes were collected from the RSD/Waste Feed Flow Loop at the beginning and end of every test. Sub-samples of these full-diversion samples are being analyzed for particle concentration and PSDs by the RJ Lee Group. This data was not received in time for inclusion in this report and will be reported in the WRPS RSD test results report. The purpose of analyzing the solids concentrations of these samples is to determine the difference between the target simulant concentration and the actual slurry concentration. The purpose of analyzing the PSD of these samples is to determine if the PSD changed during the course of the test. 



\subsection{PulseEcho Results and Discussion}

This section presents the visual and ultrasonic PulseEcho test results from the initial validation test and the formal System Performance testing in the RSD/Waste Feed Flow Loop cold-test platform.

\subsection{Validation Test Results}

The validation test for the PulseEcho instrument was the first test performed in the RSD/Waste Feed Flow Loop with the PulseEcho instrument. The purpose of the validation test was primarily to evaluate the repeatability of the PulseEcho measurements. In addition, the validation test helped ensure that PulseEcho electronics recalibration, relocation from PNNL to the Monarch test facility, and the new flow loop design at Monarch did not affect the performance of PulseEcho. The simulant selected for the validation test was 20 -wt\% broad PSD glass particles in water. This simulant was selected because PulseEcho data had been collected on it during Phase III and Phase IV testing using the MTEL at PNNL. Therefore, old data could be compared with new data to evaluate measurement repeatability.

The specifications and formulation for the broad PSD glass bead simulant was provided in Table 5.1 and Table 5.2. The dry simulant was weighed at PNNL and delivered to the Monarch test facility where the Energy Solutions operators loaded it into the RSD/Waste Feed Flow Loop. Copies of the sheets that document the weights of each dry particle component of this simulant were provided to Energy Solutions staff.

A summary of the flow velocities at which stationary particles were detected (critical velocities) for the 20 -wt $\%$ broad PSD glass beads-in-water validation test simulant is provided in Table 6.1. For easy comparison, 2012 test results obtained in the RSD/Waste Feed Flow Loop are shown with test results obtained during earlier tests at PNNL. Shown in the table are the visually detected critical velocities and the PulseEcho-detected critical velocities.

Table 6.1. Comparison of Validation Test Results from Present and 2010/11 Test Campaigns

\begin{tabular}{|c|c|c|c|c|c|}
\hline \multirow{2}{*}{ Test Campaign } & \multirow{2}{*}{ Visual $V_{\text {cr }}(\mathbf{f t} / \mathbf{s})$} & \multicolumn{2}{|c|}{$5 \mathrm{MHz}$ Transducer $\mathrm{V}_{\mathrm{cr}}(\mathrm{ft} / \mathrm{s})$} & \multicolumn{2}{|c|}{$10 \mathrm{MHz}$ Transducer $\mathrm{V}_{\mathrm{cr}}(\mathrm{ft} / \mathrm{s})$} \\
\hline & & Half Wall & Full Wall & Half Wall & Full Wall \\
\hline Phase III (2010) & 4.0 & 4.1 & $\mathrm{~N} / \mathrm{A}^{\text {(a) }}$ & $\mathrm{N} / \mathrm{A}^{(\mathrm{a})}$ & $N / A^{(a)}$ \\
\hline Phase IV (2011) & 4.0 & 4.0 & 4.0 & $\mathrm{~N} / \mathrm{A}^{(\mathrm{a})}$ & 3.9 \\
\hline Phase VI (2012) & 4.0 & $\mathrm{~N} / \mathrm{A}^{(\mathrm{a})}$ & 4.3 & $\mathrm{~N} / \mathrm{A}^{(\mathrm{a})}$ & 4.1 \\
\hline
\end{tabular}




\subsection{Validation Test Discussion}

The visually determined critical velocity for the 20 -wt $\%$ broad PSD simulant during the validation test was $4.0 \mathrm{ft} / \mathrm{s}$. This is consistent with the visually determined critical velocities for this simulant from Phase III and Phase IV testing at PNNL. During the validation test the PulseEcho system determined stationary critical velocity to be $4.1 \mathrm{ft} / \mathrm{s}$ and $4.3 \mathrm{ft} / \mathrm{s}$, as measured by the $10-\mathrm{MHz}$ and $5-\mathrm{MHz}$ transducers, respectively, at the full-wall (schedule 40 ) locations. These values are $0.1 \mathrm{ft} / \mathrm{s}$ and $0.3 \mathrm{ft} / \mathrm{s}$ higher than the visually determined critical velocity of $4.0 \mathrm{ft} / \mathrm{s}$.

The flow velocity at which particle settling was detected during the validation test by the $10-\mathrm{MHz}$ transducer at the full-wall (schedule 40) location is $0.2 \mathrm{ft} / \mathrm{s}$ higher than the corresponding Phase IV measurements. The 5-MHz validation test measurements are 0.2 to $0.3 \mathrm{ft} / \mathrm{s}$ higher than the Phase IV and Phase III measurements at the full-wall (3-in. schedule 40 pipe wall thickness) and half-wall (1/2 the pipe wall thickness of a 3-in. schedule 40 pipe) locations. Although there are differences between the Phase III/IV PulseEcho values and the validation test PulseEcho values for this simulant, the differences are not larger than ones previously obtained between visually and ultrasonically determined measurements. Therefore, the instrument's performance was found satisfactory for further testing.

\subsection{System Performance PulseEcho Test Results}

System Performance testing with the RSD/Waste Feed Flow Loop cold-test platform was conducted primarily to evaluate the RSD configuration of the Isolok ${ }^{\mathrm{TM}}$ Sampler system, but it also afforded an opportunity to continue evaluating the reliability of the ultrasonic PulseEcho instrument. A total of 17 tests were performed to evaluate the ability of the RSD Isolok ${ }^{\mathrm{TM}}$ Sampler system to obtain reliable samples from the RSD/Waste Feed Flow Loop.

This section summarizes the results from the visual observations and the PulseEcho System Performance testing. A summary of the visual and PulseEcho test results for all 17 tests is presented in Table 6.2 in the order in which the tests were performed. The PulseEcho results obtained by the 5-MHz and 10-MHz ultrasonic transducers are highlighted in green or red. Green indicates the PulseEcho instrument detected stationary particles at a flow velocity that is within $\pm 0.3 \mathrm{ft} / \mathrm{sec}$ of the visually determined flow velocities for Regime III ("stop" and "go" bed) and critical velocity V cr (stationary bed). See Bontha et al. (2010a) for more information on the definitions of the flow behavior observed during critical velocity measurements. Red indicates the PulseEcho instrument detected stationary particles at a flow velocity that is outside $\pm 0.3 \mathrm{ft} / \mathrm{s}$ of the range for Regime III and critical velocity. 
Table 6.2. Summary of Critical Velocity Detection

\begin{tabular}{|c|c|c|c|c|c|c|c|c|}
\hline \multirow[b]{2}{*}{$\begin{array}{l}\text { Test } \\
\text { Sequence }\end{array}$} & \multicolumn{3}{|c|}{ Target Simulant Properties } & \multirow[b]{2}{*}{$\begin{array}{l}\text { Regime III } \\
\text { Transitory } \\
\text { Settling }\end{array}$} & \multirow{2}{*}{$\begin{array}{c}\text { Visual } V_{c r} \\
\text { Stationary Bed } \\
\text { of Solids } \\
\text { Upstream and } \\
\underline{\text { Downstream }}\end{array}$} & \multirow[b]{2}{*}{$\begin{array}{l}\text { PulseEcho } \\
\text { Upstream } \\
\text { (5 MHz) }\end{array}$} & \multirow[b]{2}{*}{$\begin{array}{l}\text { PulseEcho } \\
\text { Downstream } \\
\text { (10 MHz) }\end{array}$} & \multirow[b]{2}{*}{ Visual Observations } \\
\hline & $\begin{array}{l}\text { Base Simulant } \\
\text { Constituents }\end{array}$ & $\begin{array}{l}\text { Supernatant } \\
\text { Simulant } \\
\text { Composition }^{(a)}\end{array}$ & $\begin{array}{l}\text { Base Simulant Mass } \\
\text { Loading/non-Newtonian } \\
\text { Bingham Yield Stress }\end{array}$ & & & & & \\
\hline 46 & Typical & Low & $9 \mathrm{wt} \%$ & Directly to $\mathbf{V}_{\mathbf{c r}}$ & 4.7 & 4.6 & 4.0 & Motion seen upstream at 4.0 and $3.9 \mathrm{ft} / \mathrm{s}$. \\
\hline 32 & Typical & Typical & $9 \mathrm{wt} \%$ & $3.6 \rightarrow 2.7$ & 2.6 & 3.4 & 3.3 & $\begin{array}{l}\text { Sliding piles of solids (dunes) started at } \\
3.4 \mathrm{ft} / \mathrm{s} \text {. Upstream settling at } 2.7 \mathrm{ft} / \mathrm{s} \text {. }\end{array}$ \\
\hline 33 & Typical & High & $9 \mathrm{wt} \%$ & $4.6 \rightarrow 4.3$ & 4.2 & 4.2 & 4.1 & Upstream settling at $4.3 \mathrm{ft} / \mathrm{s}$. \\
\hline 34 & Typical & Low & $13 \mathrm{wt} \%$ & $5.7 \rightarrow 5.2$ & 5.0 & 5.1 & 4.8 & Upstream settling at $5.2 \mathrm{ft} / \mathrm{s}$. \\
\hline 35 & Typical & Typical & $13 \mathrm{wt} \%$ & $3.7 \rightarrow 2.7$ & 2.6 & 3.5 & 3.4 & Sliding piles of solids seen at $3.5 \mathrm{ft} / \mathrm{s}$. \\
\hline 36 & Typical & High & 13 wt\% & $4.8 \rightarrow 4.4$ & 4.3 & 4.4 & 4.3 & Upstream settling at $4.5 \mathrm{ft} / \mathrm{s}$. \\
\hline 37 & High & Low & $9 \mathrm{wt} \%$ & $7.1 \rightarrow 6.9$ & 6.8 & 6.9 & 6.9 & Upstream settling at $7.0 \mathrm{ft} / \mathrm{s}$. \\
\hline 38 & High & Typical & $9 \mathrm{wt} \%$ & $5.4 \rightarrow 5.2$ & 5.1 & 5.3 & 5.0 & Upstream settling at $5.3 \mathrm{ft} / \mathrm{s}$. \\
\hline 39 & High & High & $9 \mathrm{wt} \%$ & $4.4 \rightarrow 4.1$ & 4.0 & 4.1 & 3.9 & Upstream settling at $4.1 \mathrm{ft} / \mathrm{s}$. \\
\hline 40 & High & Low & 13 wt $\%$ & $7.6 \rightarrow 7.1$ & 7.0 & 7.1 & 7.1 & Upstream settling at $7.1 \mathrm{ft} / \mathrm{s}$. \\
\hline 41 & High & Typical & $13 \mathrm{wt} \%$ & $5.6 \rightarrow 5.5$ & 5.4 & 5.4 & 5.3 & Upstream settling at $5.5 \mathrm{ft} / \mathrm{s}$. \\
\hline $41 \mathrm{a}$ & High & Typical & $13 \mathrm{wt} \%$ & $5.9 \rightarrow 5.5$ & 5.4 & 5.5 & 5.4 & Upstream settling at $5.5 \mathrm{ft} / \mathrm{s}$. \\
\hline 42 & High & High & $13 \mathrm{wt} \%$ & $4.5 \rightarrow 4.3$ & 4.2 & 4.3 & 4.1 & Upstream settling at $4.3 \mathrm{ft} / \mathrm{s}$. \\
\hline $42 a$ & High & High & $13 w t \%$ & $6.0 \rightarrow 4.2$ & 4.1 & 4.1 & 4.0 & Upstream settling at $4.2 \mathrm{ft} / \mathrm{s}$. \\
\hline 43 & & Non-Newtonian & $3 \mathrm{~Pa}^{\mathrm{a}}$ & $6.0 \rightarrow 5.1$ & 5.0 & 5.4 & $5.1^{(a)}$ & Upstream settling at $5.1 \mathrm{ft} / \mathrm{s}$. \\
\hline 44 & & Non-Newtonian & $10 \mathrm{~Pa}^{\mathrm{a}}$ & $6.8 \rightarrow 5.3$ & 5.2 & 5.7 & $5.3^{(b)}$ & \\
\hline 45 & Typical & Typical & $\begin{array}{l}13 \mathrm{wt} \% \text { ( } 5 \mathrm{wt} \% \text { of the solids } \\
\text { included as spike particles) }\end{array}$ & $3.8 \rightarrow 3.5$ & 3.4 & 3.4 & 3.5 & $\begin{array}{l}\text { Piles of solids settling and eroding at } 3.5 \\
\text { and } 3.6 \mathrm{ft} / \mathrm{s} \text {. }\end{array}$ \\
\hline \multicolumn{9}{|c|}{$\begin{array}{l}\text { (a) The } 10-\mathrm{MHz} \text { transducer did not constantly detect scattering at } 7.2 \mathrm{ft} / \mathrm{s} \text { and reported sediment more than } 10 \% \text { of the time at this flow velocity. } \\
\text { (b) The } 10-\mathrm{MHz} \text { transducer did not constantly detect scattering at } 8.3 \mathrm{ft} / \mathrm{s} \text { and reported sediment more than } 10 \% \text { of the time at this flow velocity. }\end{array}$} \\
\hline \multicolumn{9}{|c|}{ Indicates that PulseEcho instrument detected stationary particles at a flow velocity is within $\pm 0.3 \mathrm{ft} / \mathrm{s}$ of the range for Regime III and $V_{\mathrm{cr}}$} \\
\hline \multicolumn{9}{|c|}{ Indicates that PulseEcho instrument detected stationary particles at a flow velocity is outside $\pm 0.3 \mathrm{ft} / \mathrm{s}$ of the range for Regime III and $\mathrm{V}_{\mathrm{cr}}$} \\
\hline
\end{tabular}




\subsection{System Performance PulseEcho Discussion}

The objective of the PulseEcho System Performance tests in the RSD loop was consistent with the objective of prior tests at PNNL; that is., to continue to verify the reliability of the PulseEcho instrument against visual detection of stationary particles. Similar to the testing at PNNL during 2011, two different types of PulseEcho transducers - $5 \mathrm{MHz}$ sensitive to particles $>30 \mu \mathrm{m}$ and $10 \mathrm{MHz}$ sensitive to particles $>14 \mu \mathrm{m}$ - were evaluated in the RSD loop.

Several different Energy Solutions operators performed and recorded the visual observations and operated the high-resolution camera. The PNNL staff member who trained the Energy Solutions operators on the test procedure for determining critical velocity reviewed the camera files. The flow velocities at which stationary solids were determined by PNNL and Energy Solutions were within $0.1 \mathrm{ft} / \mathrm{s}$.

In general, the flow velocities at which the PulseEcho instrument detected and reported stationary particles in the RSD/Waste Feed Flow Loop are within $0.2 \mathrm{ft} / \mathrm{s}$ of the flow velocities at which stationary particles were detected visually/optically in both visual sections $\left(\mathrm{V}_{\mathrm{cr}}\right)$. In some cases the PulseEcho instrument detected and reported stationary solids in Regime III, a condition that precedes the formation of a stationary bed of particles and is characterized by transitory stationary particles or pulsatory migration of particle accumulations in the piping. For example, migrating piles of stationary solids were visually observed and ultrasonically detected by the PulseEcho instrument during Test Sequence \# 35 and Test Sequence \#32 at flow velocities that are significantly higher than the critical velocity $\left(\mathrm{V}_{\mathrm{cr}}\right)$. These migrating "stop/go" piles were stationary for a sufficient period of time and detected by the PulseEcho transducers $\geq 10 \%$ of the time during the data-acquisition window of $\geq 2.5$ minutes, which resulted in reporting stationary solids at these higher flow velocities. Plots of \% measurement indications of sediment vs. flow velocity are shown in Figure 6.1 and Figure 6.2 for Test Sequence \#35 and Figure 6.3 and Figure 6.4 for Test \#32. The 10-percent detection-reporting criterion is represented by the horizontal red line. 


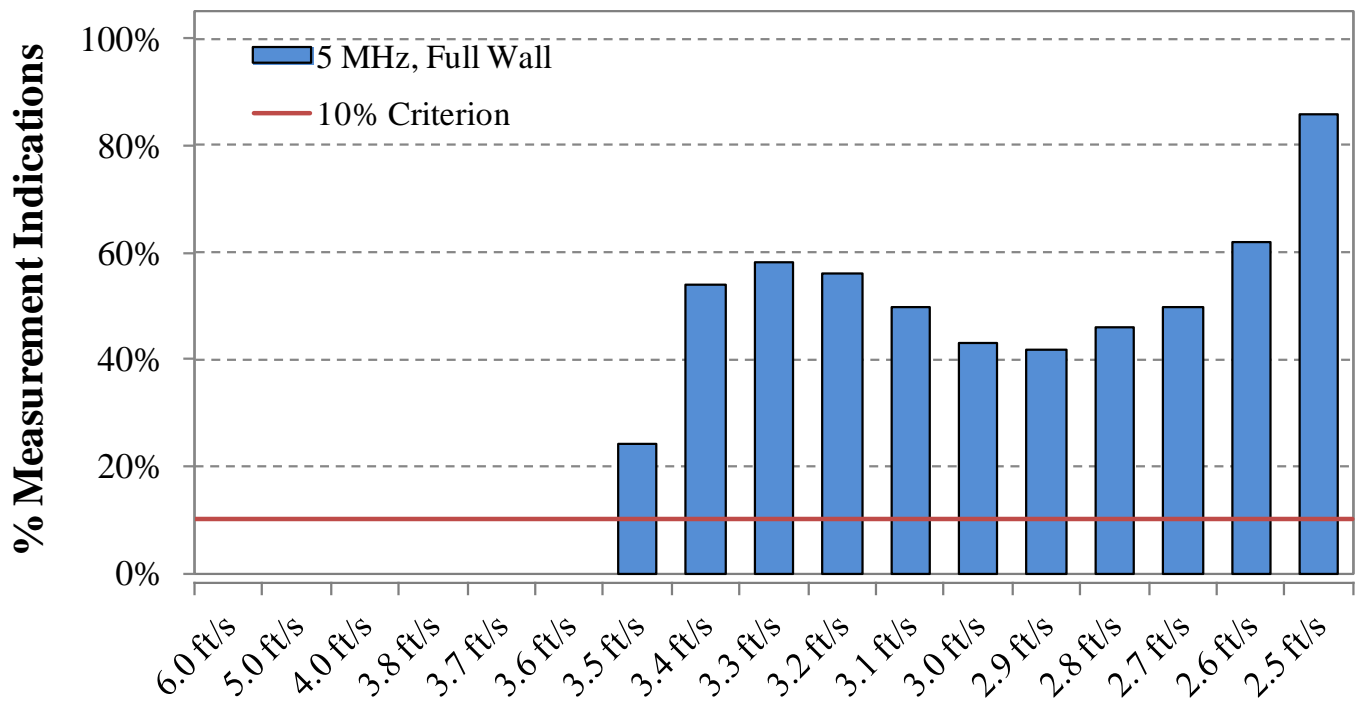

Flow Velocity Test Sequence $\rightarrow$

Figure 6.1. 5-MHz, Test Sequence \#35 (13-wt\% Solids)

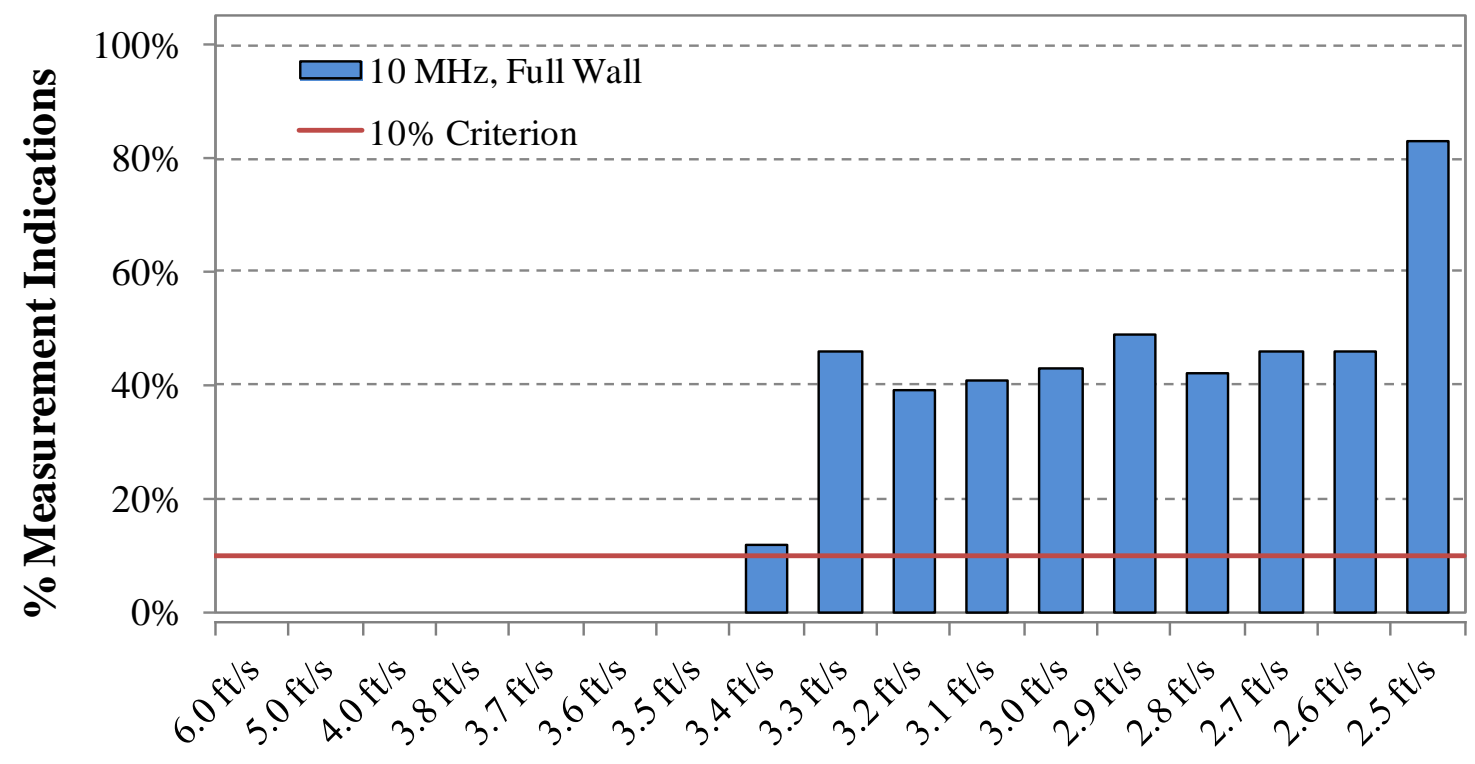

Flow Velocity Test Sequence $\rightarrow$

Figure 6.2. 10-MHz, Test Sequence \#35 (13-wt\% Solids) 


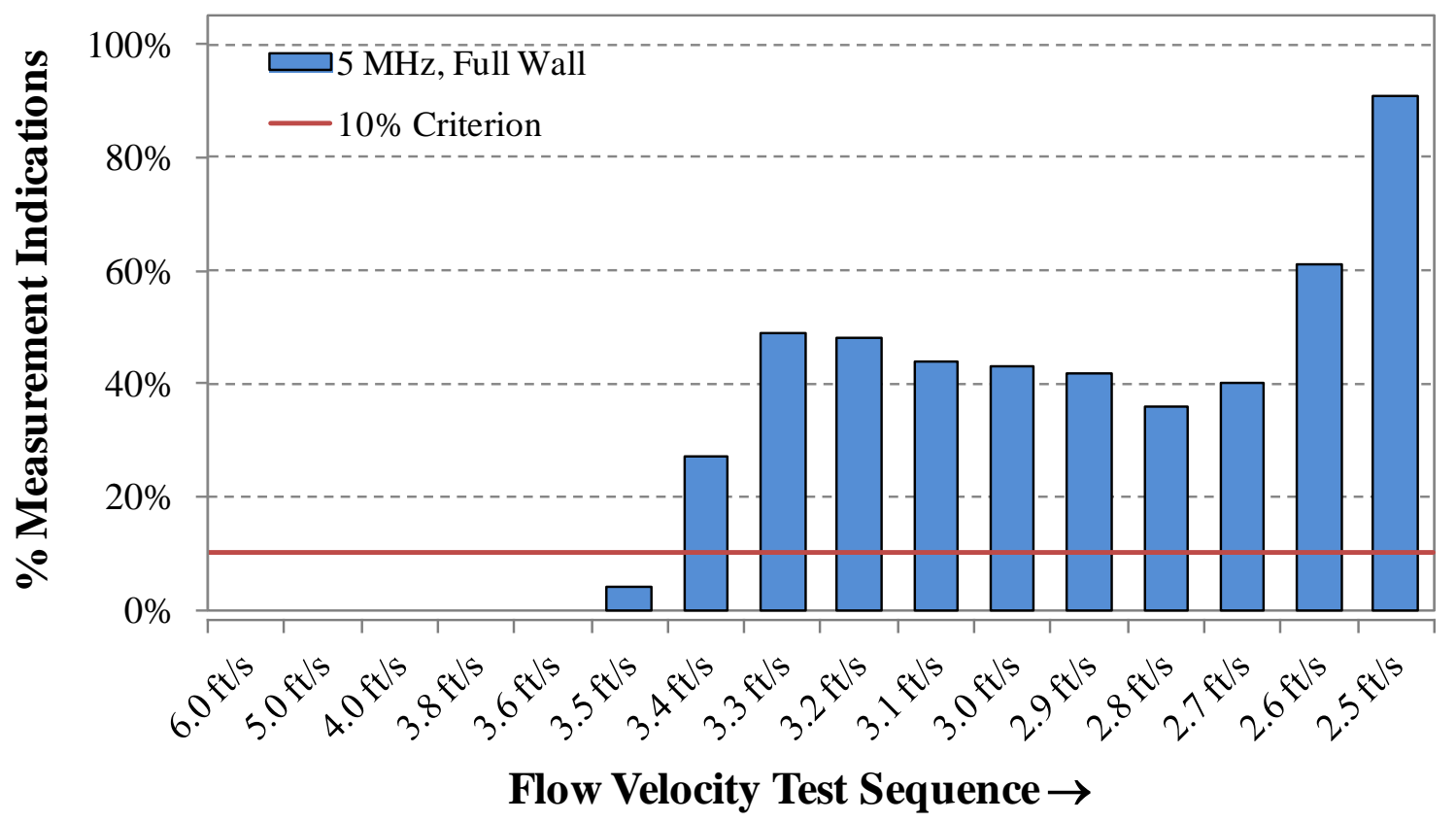

Figure 6.3. 5-MHz, Test Sequence \#32 (9-wt\% Solids)

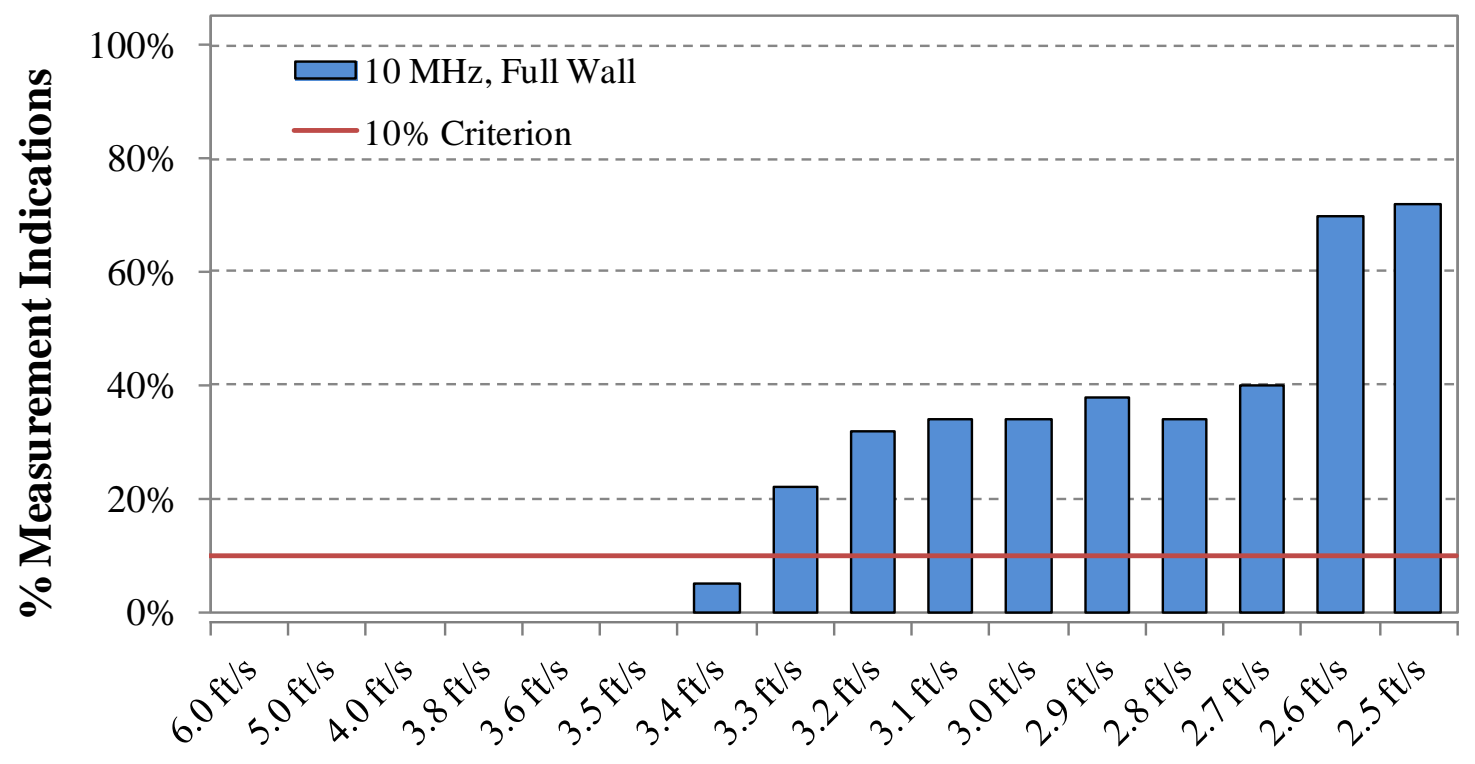

Flow Velocity Test Sequence $\rightarrow$

Figure 6.4. $10-\mathrm{MHz}$, Test Sequence \#32 (9-wt\% Solids)

The simulants used during Test Sequences \#35 and \#32 demonstrated transient particle settling (Regime III) over a wide range of flow velocities. This settling behavior emphasizes the importance of determining if this type of transitory settling will be acceptable during waste transfer to WTP. 
The discrepancy between the 5-MHz and 10-MHz transducer readings can likely be explained by the apparent settling gradient inside the PulseEcho test section. During most of the 17 tests conducted, stationary solids were observed in the upstream visual section before they were observed in the downstream visual section, indicating a stationary solids gradient was present inside the $\sim 24$-in.-long PulseEcho test section that was located between the visual test sections. The 5-MHz transducer was located 6 in. upstream of the 10-MHz transducer in the PulseEcho test section and typically detected stationary solids before the $10-\mathrm{MHz}$ transducer. This upstream-to-downstream trend in detection is consistent with the visual observations. The exact reason for this gradient is not known at the present time but it is speculated that this may be due to slight flow disturbances caused by the misalignment of the stainless steel spool and the downstream the visual section.

Test Sequence \#41 was repeated and identified as Test Sequence \#41a. The original test had a missing constituent while the repeat test had all constituents present. The visually determined critical velocity for Test Sequence \#41 and Test Sequence \#41a are the same at $5.4 \mathrm{ft} / \mathrm{s}$. The PulseEcho results also are very similar at $5.4 \mathrm{ft} / \mathrm{s}$ and $5.3 \mathrm{ft} / \mathrm{s}$ for Test Sequence \#41 and $5.5 \mathrm{ft} / \mathrm{s}$ and $5.4 \mathrm{ft} / \mathrm{s}$ for Test Sequence \#41a.

The simulant used in Test Sequence \#42 was the same as that used in the repeat test identified as Test Sequence \#42a. The original test with this simulant was repeated because there were technical difficulties with the Isolok ${ }^{\mathrm{TM}}$ sampler during the first test. However, it also afforded a repeat test with the PulseEcho instrument. The visually determined critical velocities for Test Sequence \#42 and Test Sequence \#42a are $4.2 \mathrm{ft} / \mathrm{s}$ and $4.1 \mathrm{ft} / \mathrm{s}$, respectively. The PulseEcho instrument detected settling at $4.3 \mathrm{ft} / \mathrm{s}$ and $4.1 \mathrm{ft} / \mathrm{s}$ during Test Sequence \#42 and $4.1 \mathrm{ft} / \mathrm{s}$ and $4.0 \mathrm{ft} / \mathrm{s}$ for Test Sequence \#42a. These measurements are within the $\pm 0.1 \mathrm{ft} / \mathrm{s}$ uncertainty associated with visual observations and the typical $\pm 0.2 \mathrm{ft} / \mathrm{s}$ uncertainty associated with the PulseEcho measurements.

In only one test case did a PulseEcho transducer detect stationary particles at a flow velocity that was more than $0.3 \mathrm{ft} / \mathrm{s}$ below the range for Regime III and the critical velocity. During Test Sequence \#46, the $10-\mathrm{MHz}$ transducer detected settling at $4.0 \mathrm{ft} / \mathrm{s}$ while the $5-\mathrm{MHz}$ detected settling at $4.6 \mathrm{ft} / \mathrm{s}$ and the visually determined critical velocity was $4.7 \mathrm{ft} / \mathrm{s}$. It is unclear whether the motion detected at the $10 \mathrm{MHz}$ transducer location was a reflection of actual flow conditions at the transducer location or a difference in sensitivity between the two transducer frequencies. The pre-test critical velocity was determined for Test Sequence \#46 as $3.6 \mathrm{ft} / \mathrm{s}$ while the actual critical velocity was determined to be $4.7 \mathrm{ft} / \mathrm{s}$. Test Sequence \#46 had a $1.1 \mathrm{ft} / \mathrm{s}$ difference between the pre-test critical velocity and the actual critical velocity, which is the largest difference of all the tests. The other tests typically had differences of only 0.0 to $0.3 \mathrm{ft} / \mathrm{s}$. Test Sequence \#34, which is the same simulant combination at a higher mass loading, had the second-to-largest difference of $0.6 \mathrm{ft} / \mathrm{s}$ between the pre-test and actual test critical velocity. This $1.1 \mathrm{ft} / \mathrm{s}$ difference for Test Sequence \#46 is significant, and may indicate complex flow or settling conditions for this simulant. The PulseEcho data were reviewed twice for this test sequence and modulation was present in the ultrasonic signals from the $10-\mathrm{MHz}$ transducer until $4.0 \mathrm{ft} / \mathrm{s}$, indicating particle motion was detected at that location until $4.0 \mathrm{ft} / \mathrm{s}$. The video files for this test sequence show a bed of stationary particles was present in the downstream visual section at $4.7 \mathrm{ft} / \mathrm{s}$; however, at $4.0 \mathrm{ft} / \mathrm{s}$ the entire sediment bed is eroded and then re-deposited. This phenomenon could possibly indicated nonuniform settling in the PulseEcho test section beyond the typical upstream-to-downstream gradient. The slurry constituents used in the test slurry are not different from those used in several other test sequences and do provide an explanation for the difference in measurements between the transducers. Thus, even though the $10 \mathrm{MHz}$ transducer did not detect a bed when it was observed at $4.7 \mathrm{ft} / \mathrm{s}$, there is enough 
uncertainty about both the timing and the uniformity of the settling behavior that it is difficult to assess whether or not the $10 \mathrm{MHz}$ transducer failed to reflect the in-pipe conditions.

Test Sequences \#43 and \#44 were performed with non-Newtonian, kaolin-based supernatant fluids (carrier fluids). The composition of these two test slurries only differed in the concentration of kaolin in the supernatant fluid and the resulting yield stresses. Both test slurries contained zirconium oxide particles (having a PSD d50 of 6 micron) and stainless steel particles (having a PSD d50 of 112 micron). The zirconium oxide particles are too small to provide back-scatter to either transducer, but the stainless steel particles are large enough to provide back-scatter to both transducers. The solids concentration that was detectable in each test slurry was approximately $2.5 \mathrm{wt} \%$ for both transducers. However, during Test Sequences \#43 and \#44 the 10-MHz transducer did not consistently detect particle back-scattering at the highest flow velocities of $7.2 \mathrm{ft} / \mathrm{s}$ and $8.3 \mathrm{ft} / \mathrm{s}$, respectively. The low back-scatter detected by the $10-\mathrm{MHz}$ at these high flow velocities led to the report of sediment at these high flow velocities. The 5-MHz transducer detected back-scattering at all flow velocities and did not have reports of sediment at high flow velocities during all 17 tests. The graphs showing \% sediment detection vs. flow velocity for these two tests are shown in Figure 6.5 thru Figure 6.8. Low particle back-scattering at high flow velocities for relatively low concentration slurries is a known phenomenon and has been reported and discussed in Bontha et al. (2010a) and Denslow et al (2011).

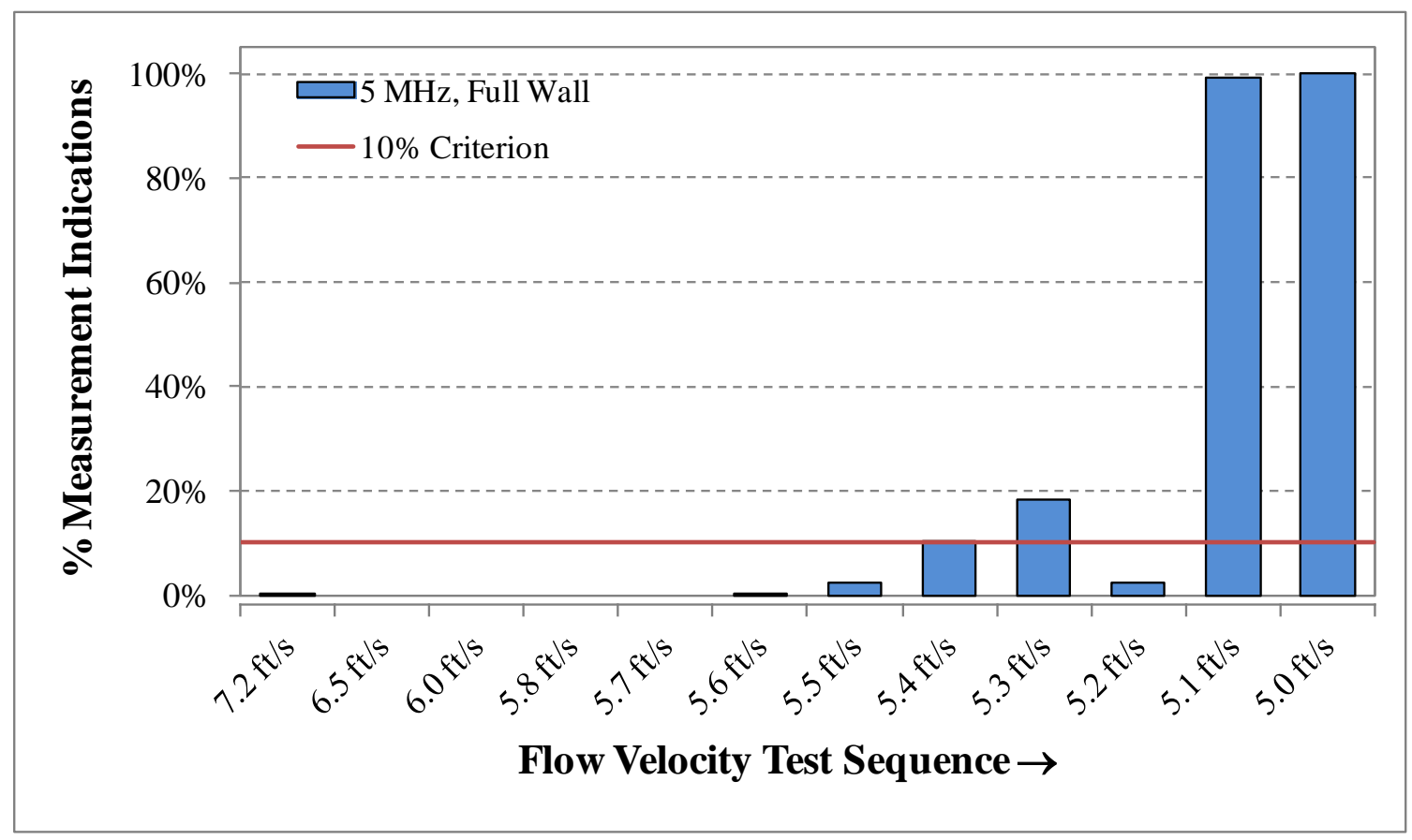

Figure 6.5. 5-MHz, Test Sequence \#43 (non-Newtonian, $3 \mathrm{~Pa}$ ) 


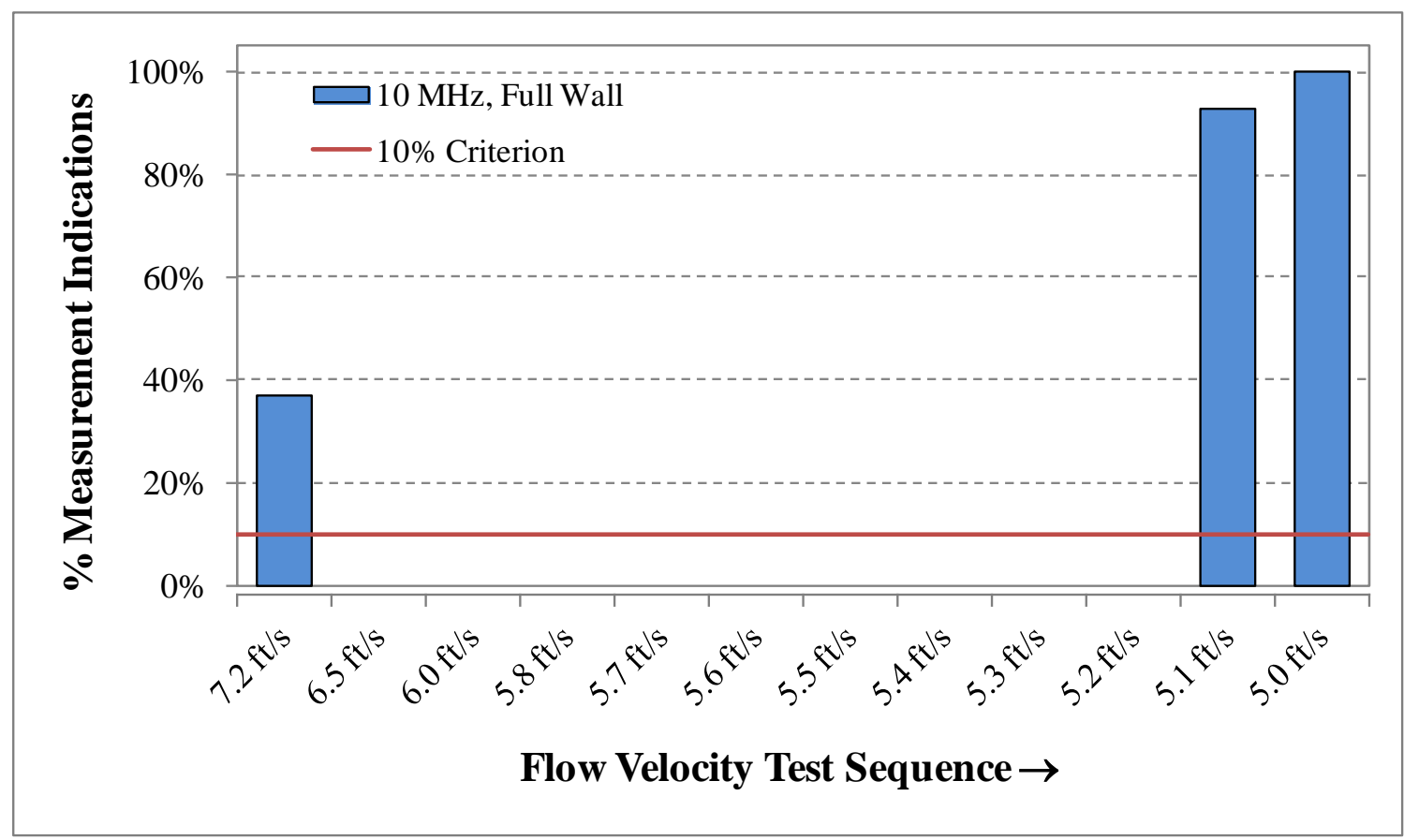

Figure 6.6. $10-\mathrm{MHz}$, Test Sequence \#43 (Non-Newtonian, $3 \mathrm{~Pa}$ )

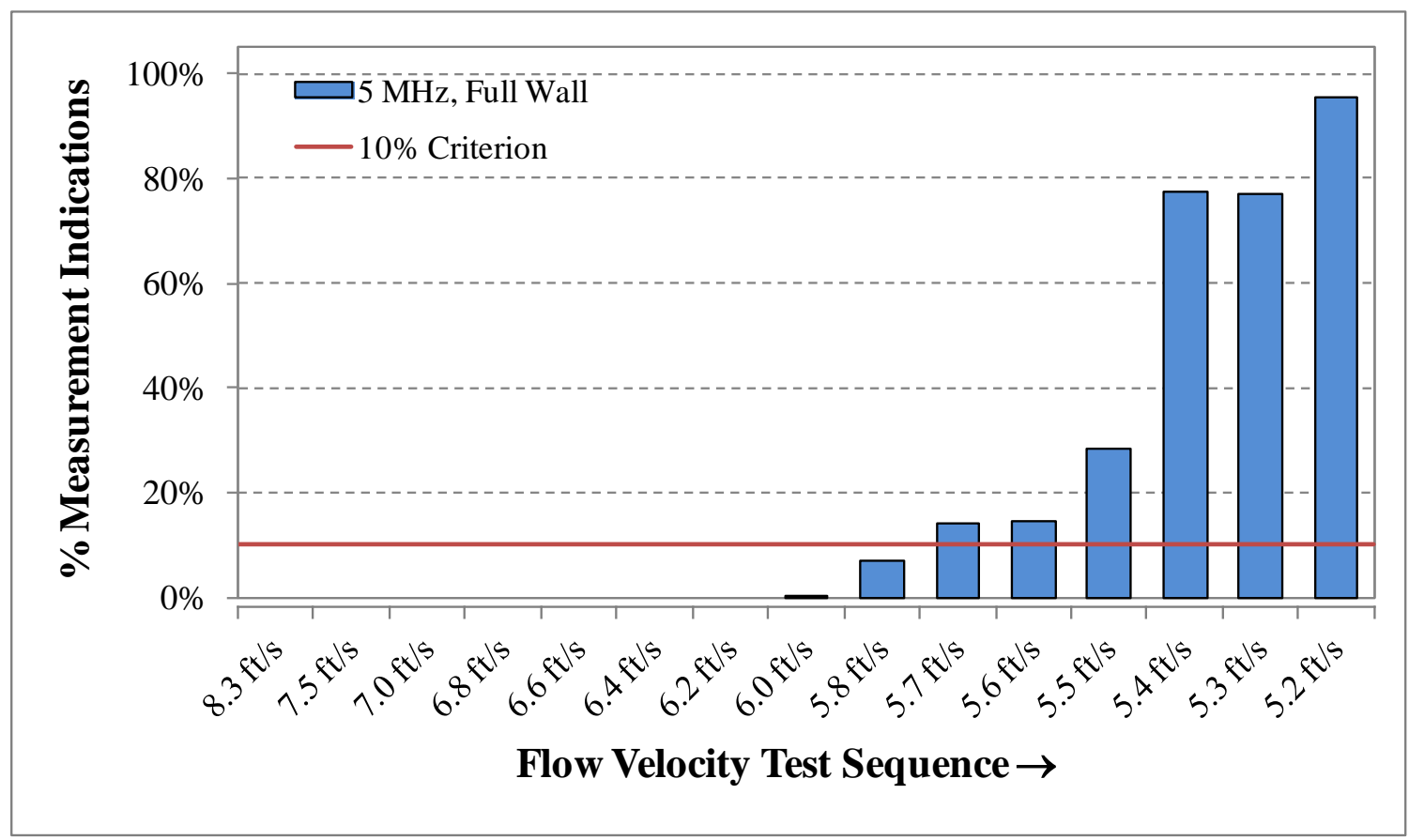

Figure 6.7. 5-MHz, Test Sequence \#44 (Non-Newtonian, $10 \mathrm{~Pa}$ ) 


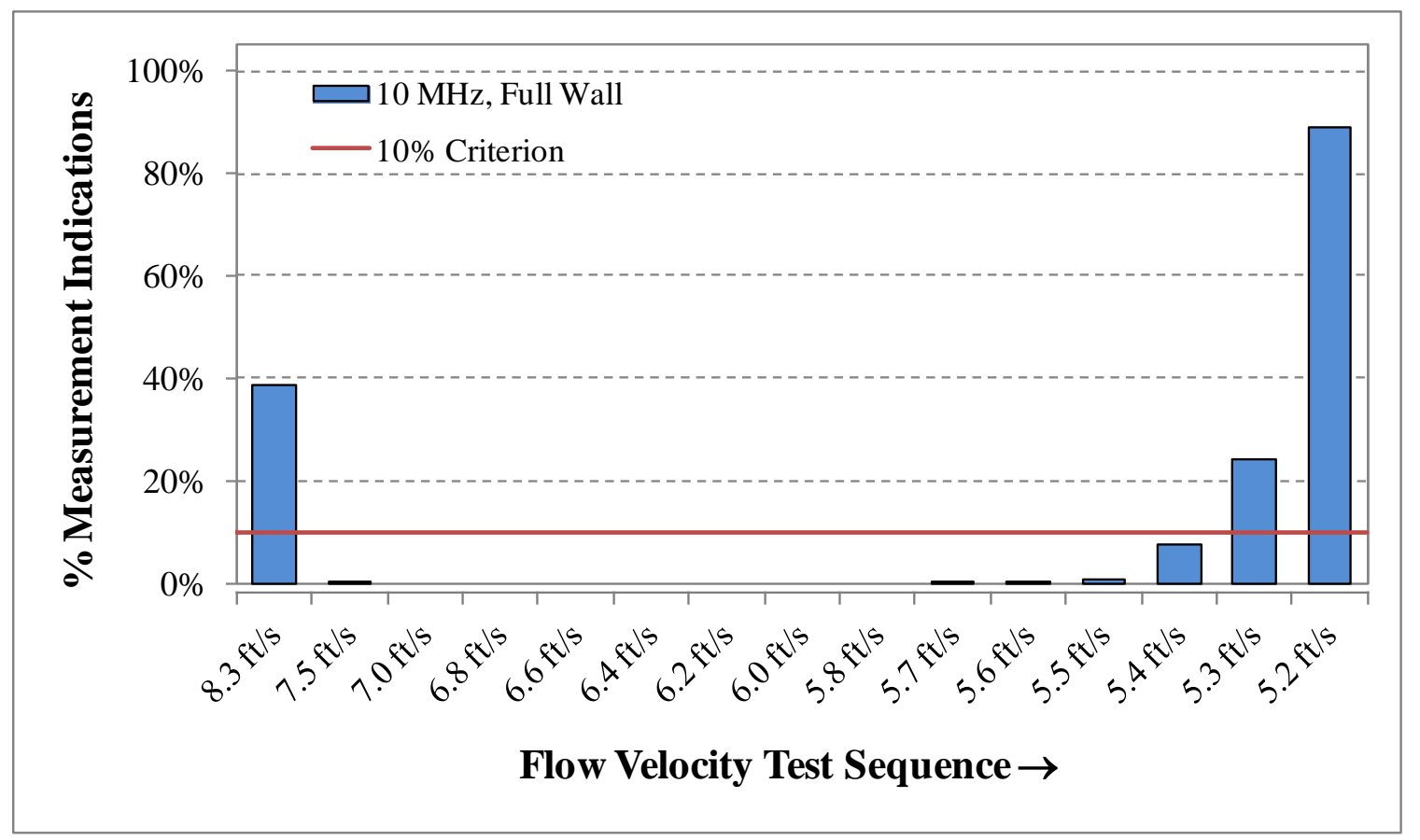

Figure 6.8. $10-\mathrm{MHz}$, Test Sequence \#44 (Non-Newtonian, $10 \mathrm{~Pa}$ )

Few slurry simulants formulated for the System Performance tests had critical flow velocities that were $\leq 4.0 \mathrm{ft} / \mathrm{s}$, and two slurry simulants had critical flow velocities near $7 \mathrm{ft} / \mathrm{s}$. However, differences between the target mass fraction of solids listed in Table 5.3 and the actual mass fraction of solids of the slurry simulants that entered the RSD/Waste Feed Flow Loop are expected, particularly if mixing and/or transfer capacity in the test loop were limited. In addition, vertical solids concentration gradients may have existed within the flow loop mixing and feed vessel that could have led to higher concentration slurries entering the flow RSD/Waste Feed Flow Loop. To quantify the exact composition of the waste stream that passed in front of the PulseEcho transducers during testing, full-diversion samples of 3-4-gal volumes were collected from the RSD/Waste Feed Flow Loop at the beginning and end of every test. Sub-samples of these full-diversion samples are being analyzed for particle concentration and PSD by the RJ Lee Group. These data and its analysis are pending and will be reported in the WRPS test results report.

The validation test performed with the PulseEcho instrument in the RSD/Waste Feed Flow Loop demonstrated good measurement repeatability and established that the instrument was not affected by changes in test loops. The 17 PulseEcho System Performance tests demonstrated that instrument could detect stationary particles for simulated Hanford Tank Farm slurries at flow velocities that were within 0.1 to $0.2 \mathrm{ft} / \mathrm{s}$ of the flow velocities at which stationary particles were detected visually/ optically for the majority of the tests. These tests continue to increase confidence in the instrument's performance and its potential for field deployment. 


\subsection{Considerations for Field Deployment}

To establish the reliability of the ultrasonic PulseEcho instrument in detecting stationary particles for determining critical flow velocity in the Waste Feed Flow Loop, three test campaigns have been conducted since FY 2010 with slurry simulants designed to encompass some or all Hanford tank waste properties that are expected to be encountered during waste retrieval. This section discusses field deployment considerations for the PulseEcho instrument.

\subsection{Un-steady State Conditions}

All testing thus far has been performed under steady-state flow conditions and PulseEcho measurements have been performed at locations that are 60 to 70 diameters downstream from points of developing flow in the full-scale test loops. These monitoring locations were selected to establish the reliability of the PulseEcho instrument because conditions in the test loops with well-developed flow were believed to be the most representative of slurry transfer conditions. Also, experimental observations made during the M1 loop testing for WTP (Poloski et al 2009a) and the Phase III/IV PulseEcho testing (Bontha et al. 2010a and 2010b) indicate that the settling behavior is a strong function of the mixing/transfer systems. In the actual Tank Farm Waste Feed Flow Loop, cyclic solids concentrations are expected due to the rotation of the mixer pumps. Solids concentrations may vary due to nozzle orientation during a mixing cycle, which will have a cycle time of approximately 5 minutes. Therefore, unpredictable settling conditions may be present in the Tank Farms Waste Feed Flow Loop. It is recommended that coldplatform testing be performed under prototypic conditions to understand the settling behavior of slurry solids under these conditions, the significance of the settling behavior in the flow loop, and whether it is representative of settling behavior in slurry transfer piping. Such an understanding would be used to determine the methodology and the most appropriate monitoring locations for the ultrasonic PulseEcho transducers on the Waste Feed Flow Loop. Installing PulseEcho transducers at more than one location along the Waste Feed Flow Loop would be a conservative approach to determining critical velocity for cyclic solids concentrations and unpredictable settling behavior. This can be achieved without multiple data acquisition systems. A multiplexer unit or switch box that automatically switches from one transducer to the next can be interfaced with one data acquisition system and multiple transducers.

\subsection{Transducer Installation}

The epoxy material used to install the ultrasonic transducers onto the machined flats on the underside of the PulseEcho test section is appropriate for laboratory-testing environments; however, it will not be appropriate for field deployment. A sustainable radiation-resistant epoxy or an alternative method for transducer installation that can tolerate radiation environments, wider temperature ranges, and thermal cycling needs to be identified for field deployment. The installation method also must allow for transducer replacement if necessary.

One installation concept for field deployment is to embed the transducers into the thick wall of the PulseEcho test section rather than bonding the transducers onto the machined flats. The embedded transducer installation concept was discussed with WRPS as a candidate option and considered to be viable for the Waste Feed Flow Loop. PNNL performed limited transducer modeling and sound field simulations to determine if the increased contact area between the transducer housing and the pipe wall 
would negatively impact signal propagation and directivity. simulations of an ultrasonic beam propagating from the front face of a 0.25-in.-diameter transducer through a schedule 40 stainless steel pipe wall is shown in Figure 7.1 and Figure 7.2 for a loosely embedded transducer (small gap between transducer housing and pipe wall) and a tightly embedded transducer (intimate contact between the transducer housing and pipe wall, respectively. The loosely embedded transducer represents the current installation configuration while the tightly embedded transducer represents the new candidate installation configuration. These simulations were performed using Imagine3D, Version 2.6, a commercial ultrasonic ray tracing software tool developed by UTEX Scientific Instrument, Inc.

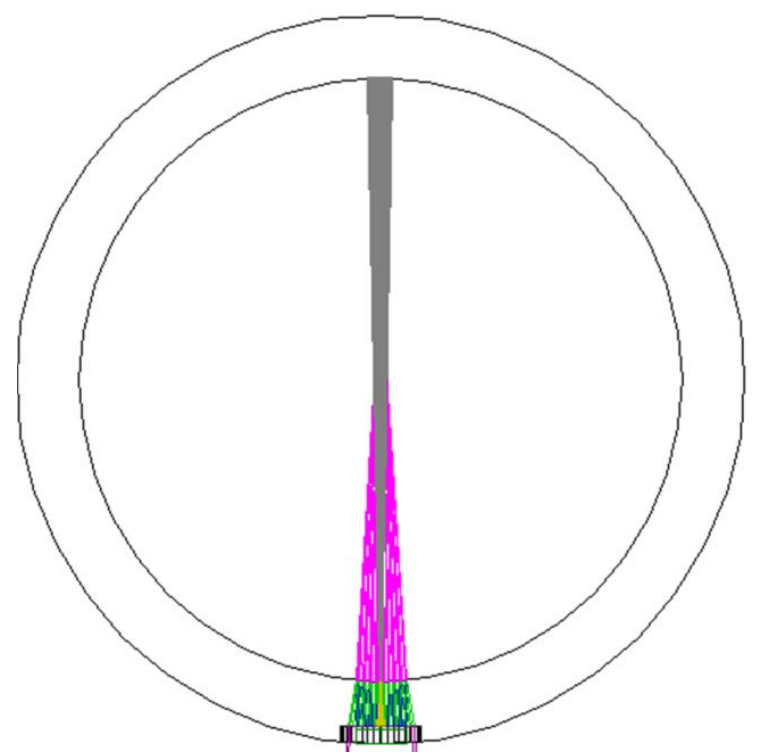

Figure 7.1. Loosely Embedded Transducer Sound Beam Simulation (current configuration)

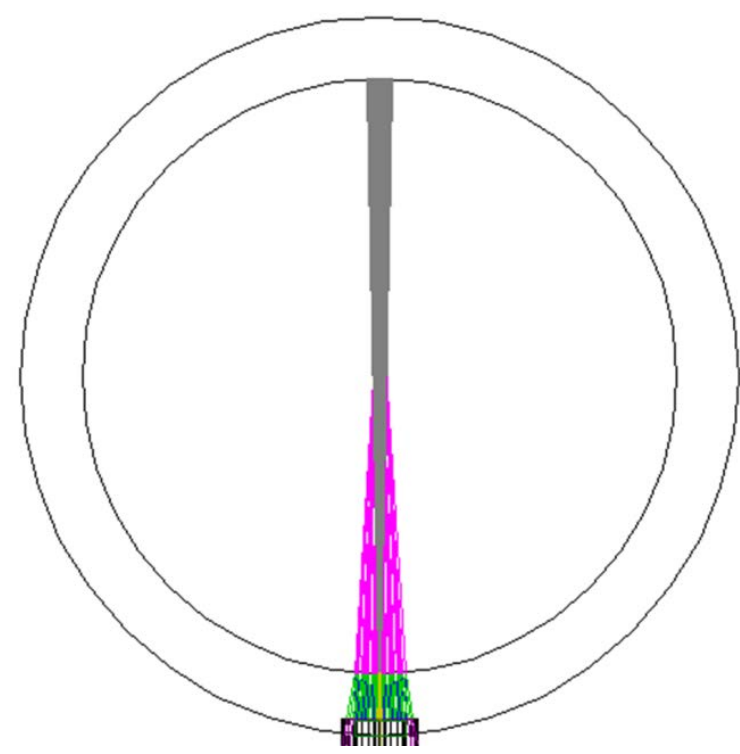


Figure 7.2. Tightly Embedded Transducer Sound Beam Simulation (new conceptual configuration)

Based on ideal transducers, there is no apparent loss in signal propagation or directivity if the contact area between the transducer and the pipe wall is increased. This configuration could be tested under a new test campaign if further testing (e.g. to evaluate oscillatory flow) is warranted.

\subsection{Radiation Hardening}

The radiation dosage tolerance of PulseEcho ultrasonic transducers, connectors, and cables needs to be evaluated to determine the expected lifetime of field-deployed transducer(s). Ultrasonic technology is routinely used in industrial nuclear environments, and ultrasonic transducers can be radiation hardened and shielded to operate in high/low temperature and low/high radiation environments. However, it is recommended that the radiation resistance of the currently utilized PulseEcho transducers be evaluated under conditions that simulate contact exposures anticipated in the field-deployed Waste Feed Flow Loop. Simulated testing would determine if the expected lifetime of the currently utilized PulseEcho transducers is sufficient or if an investment in radiation-hardened transducers is necessary. 



\subsection{Conclusions and Recommendations}

This report presents the results of 17 PulseEcho System Performance tests performed during the RSD/Waste Feed Flow Loop cold-platform testing at the Monarch test facility in Pasco, Washington. In addition, the report also presents results from a repeatability test using a simulant and test condition that was used previously in the 2010 and 2011 test campaigns. The PulseEcho System Performance testing was done using both the 5-MHz and 10-MHz ultrasonic transducers that were previously investigated at PNNL. NOTE: Although ICD-19 identifies critical velocity as a WAC, it does not define under what conditions a flow is considered to have achieved a "critical velocity". In assessing the PulseEcho technology to measure critical velocity, the definition employed by PNNL encompasses the flow velocity range between the first sign of a pulsatory or a "Stop \& Go" bed to the velocity at which a stationary bed was visually detected. The results obtained lead to the following conclusions:

1. The PulseEcho technology gives very repeatable detection of critical velocity.

2. For the majority of the tests, the PulseEcho instrument detected stationary particles at flow velocities that were within 0.1 to $0.2 \mathrm{ft} / \mathrm{s}$ of the flow velocities at which stationary particles were detected visually/optically in the RSD/Waste Feed Flow Loop.

3. In some cases, the PulseEcho instrument results in a more conservative detection of critical velocity; that is, under conditions of incipient settling as characterized by transitory stationary bed or pulsatory flow (i.e., Stop/Go) behavior at the bottom of the pipe (Regime III). Since Regime III happens before the formation of a settled bed, it is considered to be indicative of the onset of settling and PulseEcho would tend to see the "Stop/Go" motion provided its $>10 \%$ of the time.

4. For two tests, the $10-\mathrm{MHz}$ transducer did not consistently detect particle back-scattering at the highest flow velocities of $7.2 \mathrm{ft} / \mathrm{s}$ and $8.3 \mathrm{ft} / \mathrm{s}$, respectively. The low back-scatter detected by the $10-\mathrm{MHz}$ at these high flow velocities led to the report of sediment at these high flow velocities. The 5-MHz transducer, on the other hand, detected back-scattering at all flow velocities and did not have reports of sediment at high flow velocities during all 17 tests.

5. In only one test case did a PulseEcho transducer detect stationary particles at a flow velocity more than $0.3 \mathrm{ft} / \mathrm{s}$ below the range for Regime III and the critical velocity. It is unclear whether the motion detected at this sensor location was a reflection of actual flow conditions at the sensor location or a difference in particle size sensitivity between the two transducer frequencies.

6. The results from the 2012 System Performance test campaign and the 2010 Phase III and the 2011 Phase IV test campaigns continue to demonstrate the reliability and repeatability of the PulseEcho system to detect critical velocity in the actual Waste Feed Flow Loop.

All testing performed to date has been conducted under steady-state flow conditions and PulseEcho measurements have been performed at locations that are 60 to 70 pipe diameters downstream from points of developing flow in the full-scale test loops. These monitoring locations were selected because conditions in the test loops with well-developed flow eliminate uncertainties when assessing PulseEcho performance by comparison with visual measurements. In the actual Tank Farm Waste Feed Flow Loop, a set of two rotating jet mixers will be used to mix the feed vessel and the concentration of the solids drawn out through the transfer pump will vary depending on the location of the jets. Therefore, steadystate conditions in feed concentration cannot be expected to be present as the waste is pumped through the Waste Feed Flow Loop. It is not expected that the unsteady-state solids loading in the feed to the Waste 
Feed Flow loop will impact detection of a stationary bed by the PulseEcho system, but the behavior could impact the methodology used to apply the technology during actual waste feed test campaigns. In other words, based on the results obtained to date, it is believed that PulseEcho will detect the presence or absence of a stationary bed at the transducer location but translation of the PulseEcho measurement to a critical velocity will depend on 1) how the long and often measurements are made and 2) where the measurements are made. In order to address these two uncertainties in determining critical velocity, how the PulseEcho technique is implemented and used for oscillatory or transient conditions will have to be considered. Installing PulseEcho transducers at more than one location along the Waste Feed Flow Loop may be desired or necessary to ensure that settling and accumulation are detected at the points with the highest probability of settling or that have flow conditions that are representative of those that are expected to be encountered during waste transfer between the Hanford tank farms and WTP.

The two items identified above- - how the long and often measurements are made and where the measurements are made-will depend on the mixing and transfer systems used during actual waste feed transfer operations. Therefore, establishing the methodology for implementing the PulseEcho technology during cold testing of the Waste Feed Flow loop is crucial to actual field deployment of the technology. Such testing can be completed with the verified PulseEcho instrument in its current prototype state. 


\subsection{References}

Bontha JR, HE Adkins, KM Denslow, JJ Jenks, CA Burns, PP Schonewill, GP Morgen, MS Greenwood, J Blanchard, TJ Peters, PJ MacFarlan, EB Baer, and WA Wilcox. 2010a. Test Loop Demonstration and Evaluation of Slurry Transfer Line Critical Velocity Measurement Instruments. PNNL-19441, Rev.0, Pacific Northwest National Laboratory, Richland, Washington.

Bontha JR, HE Adkins, KM Denslow, JJ Jenks, CA Burns , PP Schonewill, GP Morgen, MS Greenwood, J Blanchard, TJ Peters, PJ MacFarlan, EB Baer, and WA Wilcox. 2010b. Supplementary Information for Test Loop Demonstration and Evaluation of Slurry Transfer Line Critical Velocity Measurement Instruments. PNNL-19560, Pacific Northwest National Laboratory, Richland, Washington.

Denslow KM, JR Bontha, CA Burns, NN Bauman, HE Adkins, JJ Jenks, PP Schonewill and DF Hopkins. 2011. Hanford Tank Farms Waste Certification Flow Loop Phase IV: PulseEcho Sensor Evaluation. PNNL-20350, Pacific Northwest National Laboratory, Richland, Washington.

Meyer PA, HE Adkins, KM Denslow, JA Bamberger, BE Wells, MS Greenwood, PA Scott, J Blanchard, GP Morgen. 2009. Hanford Tank Farms Waste Certification Flow Loop Test Strategy. PNNL-18688, Pacific Northwest National Laboratory, Richland, Washington.

Olson JW. 2011. ICD 19 - Interface Control Document for Waste Feed. 24590-WTP-ICD-MG-01-019, Rev 5, Bechtel National Inc., Richland, Washington.

Oroskar AR and RM Turian. 1980. "The critical velocity in pipeline flow of slurries.” AIChE Journal 26 (4) pp. 550-558.

Poloski AP, HE Adkins, J Abrefah, AM Casella, R Hohimer, F Nigl, MJ Minette, JJ Toth, JM Tingey, and SM Yokuda. 2009a. Deposition Velocities of Non-Newtonian Slurries in Pipelines. PNNL-17639, WTP-RPT-175 Rev. 0, Pacific Northwest National Laboratory, Richland, Washington.

Poloski AP, ML Bonebrake, AM Casella, MD Johnson, JJ Toth, HE Adkins, Jr, J Chun, KM Denslow, M Luna, and JM Tingey. 2009b. Deposition Velocities of Non-Newtonian Slurries in Pipelines: Complex Simulant Testing. PNNL-18316, WTP-RPT-189 Rev. 0, Pacific Northwest National Laboratory, Richland, Washington.

Smith GL and K Prindiville. 2002. Guidelines for Performing Chemical, Physical, and Rheological Properties Measurements. 24590-WTP-GPG-RTD-001 Rev. 0, Bechtel National, Inc., Richland, Washington.

Yokuda ST, AP Poloski, HE Adkins, AM Casella, RE Hohimer, NK Karri, M Luna, MJ Minette, and JM Tingey. 2009. A Qualitative Investigation of Deposition Velocities of a Non-Newtonian Slurry in Complex Pipeline Geometries. PNNL-17973, WTP-RPT-178, Rev. 0, Pacific Northwest National Laboratory, Richland, Washington. 



\section{Appendix}

\section{Test Instruction}





\section{Appendix}

\section{Test Instruction}

\begin{tabular}{|l|l|}
\hline Test Instruction Number: & TI-WRPS-003. Rev. 0 \\
\hline Effective Date: & $08 / 28 / 2012$ \\
\hline $\begin{array}{l}\text { Governing Energy } \\
\text { Solutions Procedure: }\end{array}$ & \\
\hline TITLE: & Test Instructions for Determining Steady State and Critical Velocity \\
\hline Author & \\
\hline Technical Reviewer & \\
\hline $\begin{array}{l}\text { WRPS Approval } \\
\text { (Optional) }\end{array}$ & \\
\hline
\end{tabular}




\section{AUTHORIZATIONS}

\section{Staff List and Training}

\begin{tabular}{|l|l|l|l|}
\hline $\begin{array}{l}\text { The following individuals are delegated by the Task Manager as Technical Document Reviewers for in-process } \\
\text { changes for this Test Instruction: }\end{array}$ & Signature & Initials & $\begin{array}{c}\text { Project } \\
\text { Manager } \\
\text { Approval } \\
\text { (Initials) }\end{array}$ \\
\hline \hline Printed Name & & & \\
\hline & & & \\
\hline & & & \\
\hline & & & \\
\hline & & & \\
\hline
\end{tabular}

The following staff may perform work and record data entries described in this Test Instruction.

\begin{tabular}{|l|l|l||}
\hline \hline \multicolumn{3}{|c||}{$\begin{array}{c}\text { "I read this document, discussed activities with the test lead as needed } \\
\text { and I understand the instructions." }\end{array}$} \\
\hline \hline Printed Name & Signature & \\
\hline & & \\
\hline & & \\
\hline & & \\
\hline & & \\
\hline & & \\
\hline & & \\
\hline
\end{tabular}


The following staff may perform work and record data entries described in this Test Instruction.

\begin{tabular}{|l|l|l||}
\hline \hline \multicolumn{3}{|c||}{ “I read this document, discussed activities with the test lead as needed } \\
and I understand the instructions."
\end{tabular}

\begin{tabular}{|c|c|}
\hline \multicolumn{2}{|l||}{ Provide reference to Laboratory Record Bookss that contain supporting documentation: } \\
\hline \hline Laboratory Record Book & \\
\hline & \\
\hline & \\
\hline & \\
\hline & \\
\hline & \\
\hline & \\
\hline & \\
\hline
\end{tabular}




\section{SCOPE}

This Test Instruction is intended for formal testing.

\section{GENERAL TEST APPROACH}

The purpose of this procedure is to provide instructions for determining the steady-state and pipeline critical velocities $\left(\mathrm{V}_{\mathrm{cr}}\right)$ in the test loop developed by Energy Solutions. The goal of this procedure is to ensure continuity between previous tests performed at the PNNL MTEL facility and those performed by Energy Solutions. This continuity pertains only to the method used for determining steady state and $\mathrm{V}_{\mathrm{cr}}$, anything beyond which is not covered herein.

A test will consist of flowing slurry through prototypic WRPS certification loop 3-in. pipe at velocities well above $\mathrm{V}_{\mathrm{cr}}$. Previous testing at PNNL found that starting testing at an average velocity of $8 \mathrm{ft} / \mathrm{s}$ ensured that all particles are fully suspended in the loop. Washington River Protection Solutions (WRPS) procedure TPR-RSD-OP-0001 (Rev. 1) calls for a fluid velocity of $6 \mathrm{ft} / \mathrm{s}$ at test start. However, because the simulants planned for use with the Remote Sampler Demonstration (RSD) consist of particles with sizes $>100 \mu \mathrm{m}$ and particle densities $>8 \mathrm{~g} / \mathrm{cm}^{3}$, it may be necessary to operate at higher velocities to achieve suspension. After initiating loop operation at $6 \mathrm{ft} / \mathrm{sec}$, visual observations should be made at the transparent sections. If flow behavior in Regimes II or III (i.e., a sliding or stop-go bed as discussed in Definitions section below) is observed, then the line velocity should be increased as necessary until all particles are fully suspended. The flow velocity will then be ramped down incrementally to approach $\mathrm{V}_{\mathrm{C}}$. When the flow is slightly above $V_{c r}$, the incremental velocity drop will be reduced to ensure $V_{C}$ is determined with minimal bias.

\section{KEY INFORMATION REQUIRED}

To determine steady state, the following information is required:

- Apparent flow velocity through the 3-in, pipe

- Slurry density

- Slurry temperature.

To determine $\mathrm{V}_{\mathrm{C}}$, the following information is required:

- Apparent flow velocity through the 3-in. pipe

- Visual observation on the bottom of the clear visualization sections upstream/ downstream of ultrasonic test section. A high-quality camera directed at the bottom of the visualization section is recommended to aid in determining $V_{C}$.

Before and after each test, a fluid sample will be taken for analysis. 


\section{RECORDS}

Records to be generated from implementing this Test Instruction are identified below:

- Completed Test Instruction printed and completed data sheets

- M\&TE Lists

- Laboratory Record Book entries.

\section{Materials Testing and Evaluation (M\&TE)}

An M\&TE list is to be generated prior to testing. The list will include all of the M\&TE equipment and any ancillary equipment used to support testing such as lab scales. The M\&TE is to include at a minimum the following:

- Date the configuration recorded by the M\&TE list was put in place

- Property number

- Calibration ID number

- Location of instrument (either building and room or location within test loop)

- Description of instrument (e.g. type of instrumentation)

- Manufacturer

- Manufacturer model or part number

- Serial number

- M\&TE Category per the following:

- Cat 1 M\&TE: all M\&TE calibrated externally by a qualified calibration laboratory

- Cat 2 M\&TE: all M\&TE that is user-calibrated

- Cat 3 M\&TE: commercial measuring devices that are not adjustable and provide adequate accuracy

- Cat FIO M\&TE: "For Information Only"; Cat 1 and 2 M\&TE that is not being used for quality-affecting measurement

- Date of last calibration and calibration expiration date.

In addition, either via the M\&TE list, data sheets, or LRB entries, the test record needs to track:

- Any removal of instrumentation from actual test service. Removal should include any temporary removal for cleaning or maintenance. The M\&TE list should reflect the removal from service (the test setup) even if the item is not absent for any period of testing.

- When any maintenance of instrumentation are performed unless part of normal execution of the Test Instruction.

- Any configuration change in the location or integration of the M\&TE with the test setup.

Changes/updates made to the M\&TE should be noted in an Laboratory Record Book.

\section{DEFINITIONS}

Steady State. This condition is defined by no significant change in any of the measured slurry parameters (i.e., temperature, flow, density) over time. The primary driver for defining steady state is density. When the bulk density change is less than $0.01 \mathrm{~g} / \mathrm{ml}$ over a 5-minute interval, 
steady state can be declared. Regardless, steady state should not be declared prior to a minimum 5-minute wait.

Critical Velocity Regimes. During testing the critical velocity is defined as the point at which the first solids settle along the bottom of the pipe (i.e., a narrow stationary bed forms). In general, three distinct flow regimes can be observed that occur before a stationary bed (i.e., critical velocity) is formed. Regime I (focused circumferential chaotic particle motion), Regime II (focused axial motion), Regime III (pulsatory sliding bed), and a stationary bed can be observed using a camera system directed at the bottom of the visualization section. These regimes are not always visible depending on the combination of simulant/carrier fluid used. The regimes are depicted in the figure below. Visual observation across the entire section is not possible. Therefore, $\mathrm{V}_{\mathrm{C}}$ should correspond to a stationary bed that forms across the test section as observed in both the upstream and downstream visualization sections. Detection of regimes requires the use of a high-quality digital video camera and sufficient lighting. The $\mathrm{V}_{\mathrm{C}}$ should not be confused with the deposition velocity, below which particles begin to settle and form a moving bed.

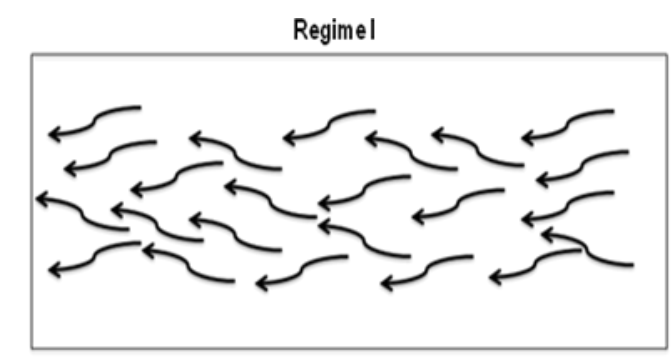

Regimell

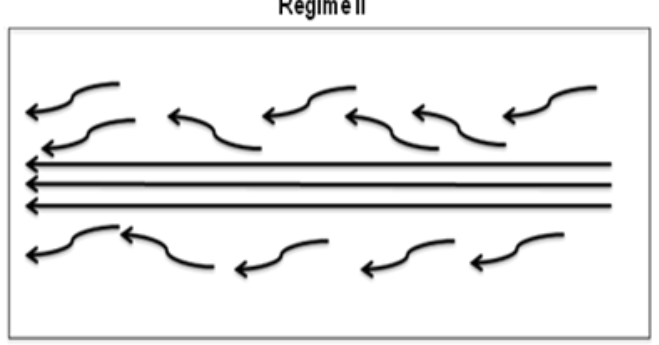

Regime III

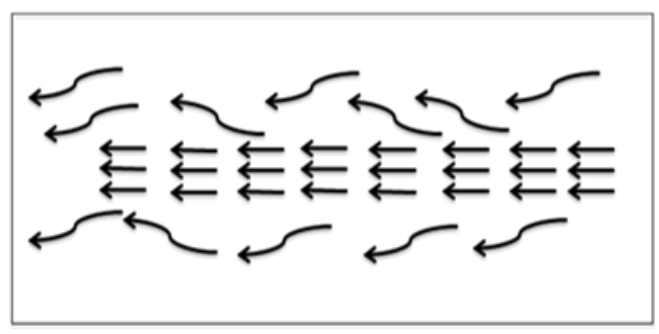

Figure A.1.. Flow Regimes Observed on Bottom of Pipe Prior to a Stationary Bed Formation

\section{TEST INSTRUCTIONS}

The approach for determining critical velocity is broken into two steps: 
1. Pre-test preparation

2. Incremental velocity sequence.

The pre-test preparation involves coarsely determining the velocity (to $\pm 0.2 \mathrm{ft} / \mathrm{s}$ ) at which a stationary bed is visually observed. Such a coarse determination of the $\mathrm{V}_{\mathrm{C}}$ helps reduce the time necessary to conduct a test. During the pre-test preparation, no PulseEcho data will be collected.

Once the $\mathrm{V}_{\mathrm{C}}$ has been coarsely determined, the particles are resuspended at the starting suspension velocity and the velocity is incrementally decreased to enable determination of the $\mathrm{V}_{\mathrm{cr}}$ to $\pm 0.1 \mathrm{ft} / \mathrm{s}$. PulseEcho measurements will be made during incremental determination of $\mathrm{V}_{\mathrm{cr}}$.

The instructions for coarse and incremental velocity sequence of testing are included in Attachments 1 and 2, respectively. These attachments (without the watermark) can be copied and reused as necessary for the different simulant tests that are planned for the present evaluation.

NOTE: The following Attachments 1 through 5 without the "SAMPLE" watermark can be printed out each day to capture data from a day's testing. Completed Attachment 1 through 5 sheets from each day's testing shall be grouped as Test Data Packages (TDPs) using the TDP cover sheet (Form 62614-QA-F1702-01_R0) located on the project share drive at: IIpnl\projects\CertLoop\Phase_6\QA_Procedures\QA Forms. 


\section{ATTACHMENT 1 - PRE-TEST PREPARATION FOR COARSE V $_{\mathrm{cr}}$ DETERMINATION}

1. _ _ Record the date and test ID number on the top of Page 8.

2. __ Confirm that the simulant has been prepared and mixed and note mixing duration:

3. __ Start flow through the loop at pre-test velocity as stated in WRPS procedure TPR-RSD-OP-0001 (Rev. 1) and ensure that steady state has been achieved.

4. __ Visually observe flow in both transparent sections to ensure that Regimes II and III or a stationary bed is not present. If none are present, proceed to Step 6.

5. _ _ If Regimes II, III, or a stationary bed are present, then increase the velocity until the particles are fully suspended after steady state has been established. Visually verify that solids have been resuspended. Record this velocity below:

Velocity for Particle Suspension:

6. __ Incrementally decrease the velocity until the point at which the bed becomes stationary in the visualization section. This may involve a few iterations that require re-suspending solids at a superficial fluid velocity at or above that determined in Step 5 and reducing the velocity to the point (within $0.2 \mathrm{ft} / \mathrm{s}$ ) that the bed becomes stationary. Note the velocity:

Pre-Test Estimated $\mathrm{V}_{\text {cr: }}$ 


\section{ATTACHMENT 2 - INCREMENTAL VELOCITY SEQUENCE FOR $\mathrm{V}_{\mathrm{cr}}$ DETERMINATION:}

\section{General Notes:}

- Prior to initiating any test, the slurry should be fully suspended and steady state achieved.

- Upon reaching steady state for each velocity, verbally communicate the data collection window is "Open." When the window is "Open," record all data at each velocity point and note any observations (such as the onset of a particular flow regime). Wait until the UPE operator indicates all data is collected, whereupon the collection window can be "Closed."

- In the Flow Loop Datasheet, note the time (and any other data), when the flow is set to a particular velocity set point "Set", when the data collection window is "open" and when it is "closed."

1. __ Sync DAS, UPE, and Camera time

2. — _ Record the date and test ID number on the top of pages 9 to 10.

3. _ _ Ensure that the slurry has been circulating in the loop for a minimum of 10 minutes at fully suspended velocity determined in Step 3 or 5 of Attachment 1. Collect video at each velocity for 10 to 15 seconds and document in Attachment 5 .

4. __ Collect a full-diversion sample per TPR-RSD-OP-0001 (Rev. 1) and MAN-RSD-OP0001 Section 3.2 (Rev. 0). Record the sample ID below: Initial Sample ID:

5. __ Incrementally drop the velocity by $1 \mathrm{ft} / \mathrm{s}$ unless the incremental drop results in surpassing $0.5 \mathrm{ft} / \mathrm{s}$ above the Pre-Test $\mathrm{V}_{\mathrm{cr}}$ (If this is the case drop to the velocity equal to $0.5 \mathrm{ft} / \mathrm{s}$ above Pre-Test $\mathrm{V}_{\mathrm{cr}}$ ). Collect video at each velocity for 10 to15 seconds and document in Attachment 5.

6. __ Upon reaching $0.5 \mathrm{ft} / \mathrm{s}$ above previously estimated Pre-Test $\mathrm{V}_{\mathrm{cr}}$, reduce velocity by $0.1 \mathrm{ft} / \mathrm{s}$ increments. Note the velocity of any regimes visually detected during the incremental drop. Collect video at each velocity for 10 to 15 seconds and document in Attachment 5.

7. _ C Continue at $0.1 \mathrm{ft} / \mathrm{s}$ incremental velocity reductions until the PulseEcho (UPE) instrument detects a stationary bed and it is also observed in both the upstream/downstream visualization sections. Note the velocity when bed is stationary in both visualization sections as $\mathrm{V}_{\mathrm{cr}}$ in the Flow Loop Datasheet. Collect video at each velocity for 10 to 15 seconds and document in Attachment 5.

8. _ _ When practical, reduce the velocity by a few $0.1 \mathrm{ft} / \mathrm{s}$ increments below the UPE detection point per UPE test operator discretion.

9. __ Re-suspend slurry at fully suspended velocity for a minimum of 10 minutes and steady state.

10. __ Upon completion of UPE testing, collect a full-diversion sample per TPR-RSD-OP0001 (Rev. 1) and MAN-RSD-OP-0001 Section 3.2 (Rev. 0).

Post-Test Final Sample ID: 
ATTACHMENT 3 - FLOW LOOP DATA SHEET

Test ID:

Testing Date:

Simulant Description:

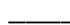

Completed by:

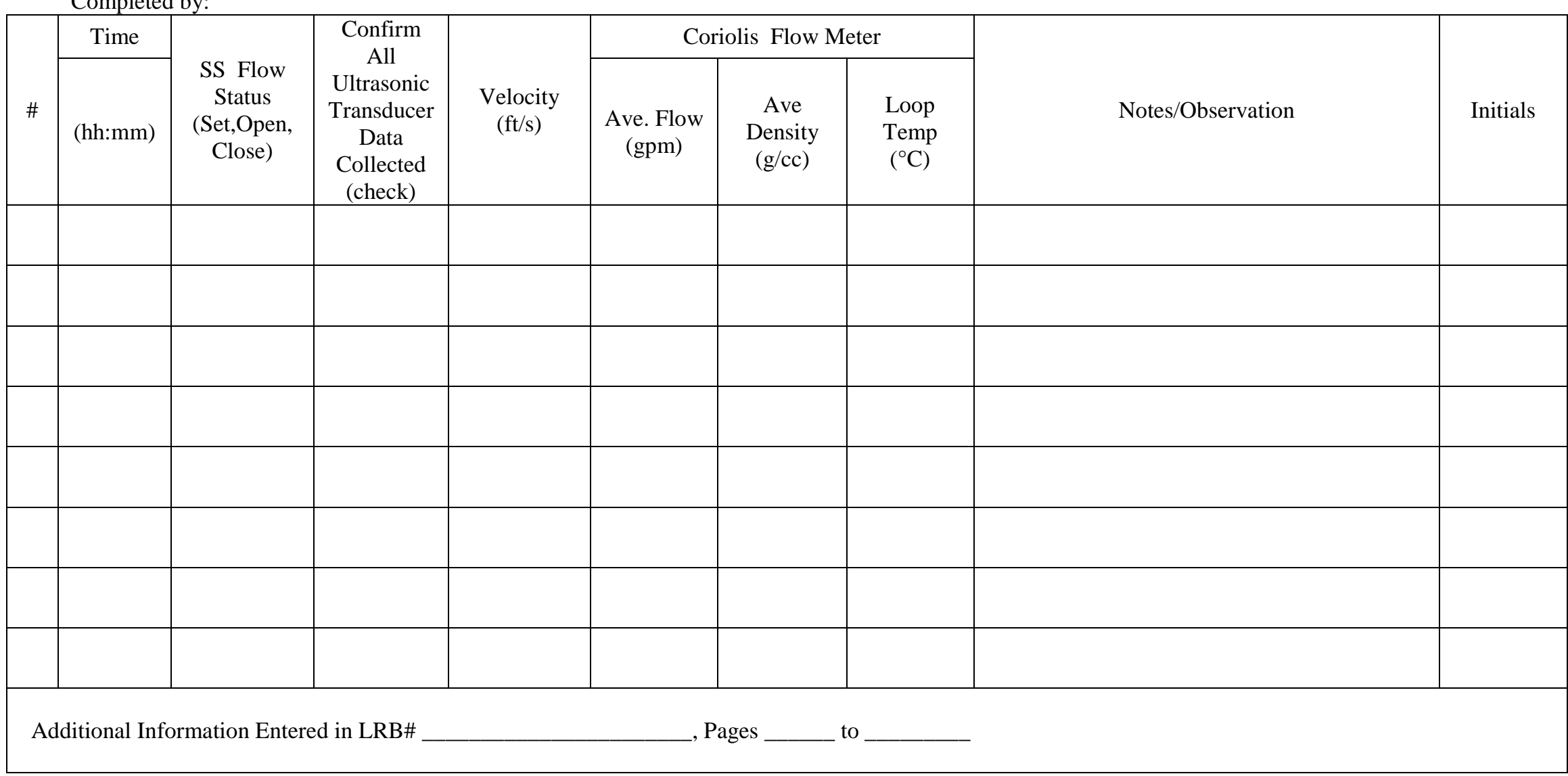

Flow loop Coriolis meter data file(s): 


\section{ATTACHMENT 4 - PULSE-ECHO DATA FILES}

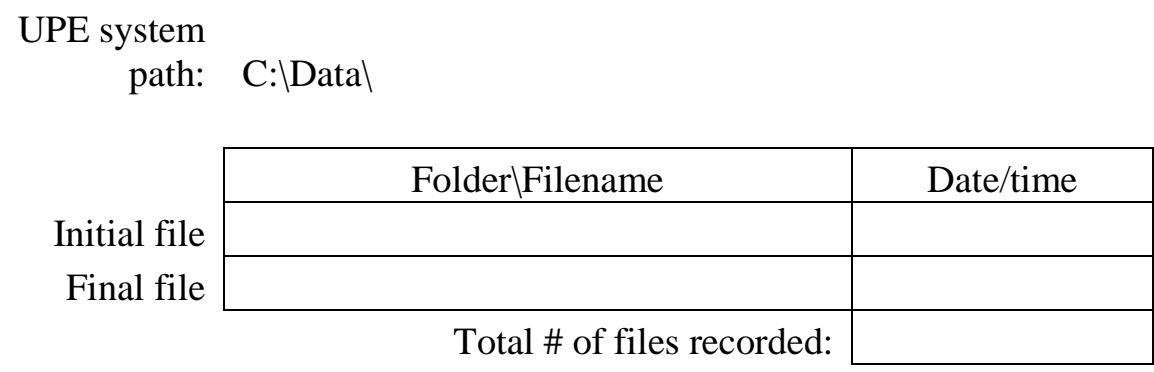

All files backed up by:

Date: 


\section{ATTACHMENT 5 - VIDEO CAMERA DATA FILES}

\begin{tabular}{|l|l|l|}
\hline Filename (TID_Velocity_HH:MM) & File Time Stamp & Length (s) \\
\hline & & \\
\hline & & \\
\hline & & \\
\hline & & \\
\hline & & \\
\hline & & \\
\hline & & \\
\hline & & \\
\hline & & \\
\hline & & \\
\hline & & \\
\hline & & \\
\hline & & \\
\hline & & \\
\hline & & \\
\hline & & \\
\hline & & \\
\hline & & \\
\hline & & \\
\hline & & \\
\hline & & \\
\hline & & \\
\hline & & \\
\hline & & \\
\hline & & \\
\hline & & \\
\hline & & \\
\hline
\end{tabular}


A.A.1 


\section{Distribution}

No. of

Copies

20 Pacific Northwest National Laboratory

KM Denslow K5-26

JR Bontha (5) P7-25

HE Adkins $\quad$ K7-15

JJ Jenks K7-15

DF Hopkins $\quad$ K8-91

BE Wells K7-15

6 Washington River Protection Solutions

M Thien (5) B1-55

S Kelly B1-55

T Wooley B1-55

2 Office of River Protection (DOE)

JS Shuen (2) H6-60

Distr.1 


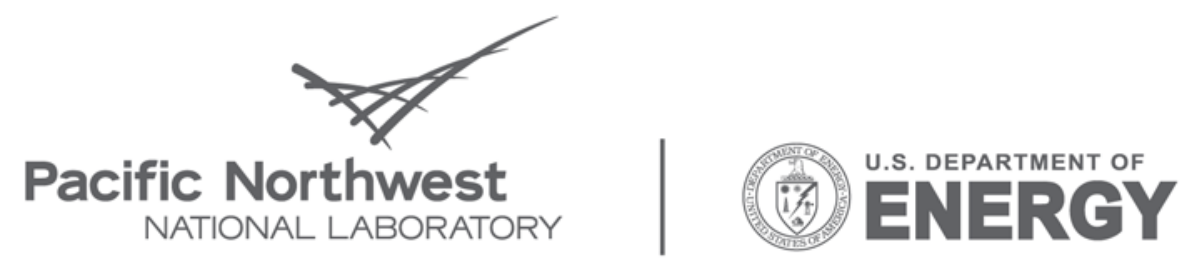

Proudly Operated by Battelle Since 1965

902 Battelle Boulevard

P.O. Box 999

Richland, WA 99352

1-888-375-PNNL (7665)

www.pnl.gov 